Rev. Int. Contam. Ambie. 36 (4) 907-925, 2020

https://doi.org/10.20937/RICA.53581

\title{
CORRELACIÓN PAN-O 3 EN EL SUROESTE DE LA CIUDAD DE MÉXICO
}

Correlation $\mathrm{PAN}_{-} \mathrm{O}_{3}$ in southwest Mexico City

\author{
Norma Irene CARRASCO-MIJAREZ ${ }^{1 *}$, Ricardo TORRES-JARDÓN ${ }^{2}$ \\ y Hugo Alberto BARRERA-HUERTAS ${ }^{3}$
}

${ }^{1}$ Programa de Posgrado en Ciencias de la Tierra. Universidad Nacional Autónoma de México. Ciudad Universitaria. 04510 Coyoacán, CdMx. México.

${ }^{2}$ Centro de Ciencias de la Atmósfera. Universidad Nacional Autónoma de México. Ciudad Universitaria. 04510 Coyoacán, CdMx. México.

${ }^{3}$ Departamento de Ingeniería en Sistemas Ambientales. Escuela Nacional de Ciencias Biológicas. Instituto Politécnico Nacional. Av. Wilfrido Massieu, Unidad Adolfo López Mateos, 07738 Gustavo A. Madero, CdMx. México.

*Autora para correspondencia: carrascomijarez@yahoo.com.mx

Palabras clave: nitrato de peroxiacetilo, contaminación fotoquímica, monitoreo atmosférico, Valle de México.

\section{RESUMEN}

Se midieron los contaminantes nitrato de peroxiacetilo (PAN) y dióxico de nitrógeno $\left(\mathrm{NO}_{2}\right.$, verdadero) durante dos campañas cortas de monitoreo en junio y octubre de 2017 con un método por cromatografía de gases y detección por luminol al suroeste de la Ciudad de México. Los promedios horarios máximo y total de la razón de mezcla medida del PAN de cada campaña fueron de 5.2 y 1.3 ppb $_{\mathrm{v}}$ en junio y de 3.3 y $0.6 \mathrm{ppb}_{\mathrm{v}}$ en octubre, respectivamente, ligeramente menores a los promedios reportados en 2003. Esto sugiere que la emisión de precursores de este contaminante: acetaldehído y butenos, han disminuido sólo parcialmente en los últimos 15 años. Los promedios horarios del PAN fueron correlacionados con $\mathrm{O}_{3}$ y $\mathrm{NO}_{2}$ (medido por quimioluminiscencia), radiación solar, temperatura, e intensidad del viento registrados en el mismo sitio para obtener un modelo empírico combinado (MEC) para el diagnóstico aproximado de las concentraciones del PAN para otros periodos del año. El MEC resultante se integró con varias correlaciones lineales múltiples que se aplican dependiendo del periodo del día y la temperatura. La correlación entre el PAN simulado y el medido para las dos campañas resultó en un coeficiente $\mathrm{R}^{2}=0.87$. Se aplicó el MEC a registros de las variables antes mencionadas de la estación de monitoreo del Centro de Ciencias de la Atmósfera en la Ciudad Universitaria para la temporada de ozono de 2017. Se observó una correlación positiva del PAN con el $\mathrm{O}_{3}$ dependiente de la temperatura, que fue cada vez más débil conforme la temperatura excedió $\operatorname{los} 20^{\circ} \mathrm{C}$.

Key words: peroxyacetyl nitrate, photochemical pollution, atmospheric monitoring, Mexico Valley.

\begin{abstract}
Peroxyacetyl nitrate (PAN) and $\mathrm{NO}_{2}$ (true) were measured during two short campaigns in June and October, 2017 with a gas chromatography-luminol detection method in southwest Mexico City. The maximum and total hourly mixing ratio measured averages of PAN were 5.2 and $1.3 \mathrm{ppb}_{\mathrm{v}}$ in June and 3.3 and $0.6 \mathrm{ppb}_{\mathrm{v}}$ in October, respectively, being these values slightly lower than those reported in 2003 suggesting that PAN precursor levels have only
\end{abstract}


partially decreased during the last 15 years. PAN hourly averages of the two campaigns were correlated with $\mathrm{O}_{3}, \mathrm{NO}_{2}$ (by chemiluminescence), solar radiation, temperature, and wind speed measured in the same monitoring site in order to obtain a combined empirical model (CEM) for the diagnosis of PAN concentrations for other periods of the year. The resulting CEM was integrated with several multiple linear correlations which are applied depending on the period of the day and the ambient temperature. The correlation between both, the simulated and measured PAN resulted in a coefficient $\mathrm{R}^{2}=0.87$. The CEM was applied to records of the above mentioned variables measured at the Centro de Ciencias de la Atmósfera monitoring station at Ciudad Universitaria for the 2017 ozone season. A positive temperature dependent correlation was obtained between PAN and ozone, but the correlation was weaker as ambient temperature exceeded the $20^{\circ} \mathrm{C}$.

\section{INTRODUCCIÓN}

Los nitratos de peroxiacilos son un grupo importante de contaminantes secundarios fotoquímicos asociados a la formación del ozono $\left(\mathrm{O}_{3}\right)$ en áreas urbanas. De ellos, el nitrato de peroxiacetilo (PAN: peroxyacetyl nitrate, $\left.\mathrm{CH}_{3} \mathrm{C}(\mathrm{O}) \mathrm{O}_{2} \mathrm{NO}_{2}\right)$ es la especie más abundante de esta familia (Singh 1987). Su importancia radica, al igual que la del ozono, en sus efectos potenciales en la salud y los ecosistemas, y en su participación indirecta en la química atmosférica regional y sinóptica. Los peroxiacilos y en particular el PAN son fuertes irritantes de los ojos, lacrimógenos y fitotóxicos (Singh 1987, Kourtidis et al. 1993). Los niveles de concentración de esta especie expresados como razón de mezcla son menores a 8 ppbv, sin embargo, se han reportado niveles de hasta varias decenas de $\mathrm{ppb}_{\mathrm{v}}$ en zonas urbanas contaminadas, incluyendo a la Ciudad de México (Singh 1987, Altshuller 1993, Rappenglück et al. 1993, Gaffney et al. 1999, Rappenglück et al. 2000). No obstante de su importancia como contaminante secundario, no es rutinaria su medición debido a las dificultades que esto implica.

$\mathrm{Ni}$ el $\mathrm{O}_{3}$ ni el PAN son emitidos por alguna fuente específica sino que son rápidamente co-producidos en la tropósfera a través de diversas reacciones fotoquímicas entre los óxidos de nitrógeno $(\mathrm{NOx}=\mathrm{NO}$ $+\mathrm{NO}_{2}$ ) y compuestos orgánicos volátiles $(\mathrm{COV})$ en presencia de luz solar (Roberts et al. 1995, Seinfeld y Pandis 2012). La secuencia siguiente ejemplifica el caso general de la oxidación de un alcano (donde $\mathrm{R}$, un grupo alquilo, representa el resto de la cadena orgánica) para producir radicales peroxílo $\left(\mathrm{HO}_{2} \mathrm{O}\right.$ $\mathrm{RO}_{2}{ }^{\circ}$ ) (NARSTO 2000).

$\mathrm{RH}+\mathrm{OH} \rightarrow \mathrm{R}^{\bullet}+\mathrm{H}_{2} \mathrm{O}$

$\mathrm{R}^{\bullet}+\mathrm{O}_{2} \rightarrow \mathrm{RO}_{2}{ }^{\circ}$

$$
\begin{aligned}
& \mathrm{RO}_{2}^{\bullet}+\mathrm{NO} \rightarrow \mathrm{RO}^{\bullet}+\mathrm{NO}_{2} \\
& 2\left\{\mathrm{NO}_{2}+\mathrm{h} v(\lambda<420 \mathrm{~nm}) \rightarrow \mathrm{NO}+\mathrm{O}\left({ }^{3} \mathrm{P}\right)\right\} \\
& 2\left\{\mathrm{O}\left({ }^{3} \mathrm{P}\right)+\mathrm{O}_{2}+\mathrm{M} \rightarrow \mathrm{O}_{3}+\mathrm{M}\right\} \\
& \mathrm{RO}^{\bullet}+\mathrm{O}_{2} \rightarrow \text { compuesto carbonilo }+\mathrm{HO}_{2}{ }^{\circ} \\
& \mathrm{HO}_{2}{ }^{\bullet}+\mathrm{NO} \rightarrow \mathrm{OH}+\mathrm{NO}_{2}
\end{aligned}
$$

Neta: $\mathrm{RH}+4 \mathrm{O}_{2}+2 \mathrm{~h} v \rightarrow \mathrm{H}_{2} \mathrm{O}+2 \mathrm{O}_{3}+$ carbonilo

Donde el carbonilo generalmente es un aldehído como formaldehído, acetaldehído o acetona. El PAN por su parte, es producido por la reacción reversible del radical peroxiacetilo $\left(\mathrm{CH}_{3} \mathrm{C}(\mathrm{O}) \mathrm{OO}{ }^{\circ}\right)$ con $\mathrm{NO}_{2}$ (Fischer et al. 2014):

$$
\mathrm{CH}_{3} \mathrm{C}(\mathrm{O}) \mathrm{OO}^{\bullet}+\mathrm{NO}_{2}+\mathrm{M} \rightarrow \mathrm{PAN}+\mathrm{M}
$$

Donde $\mathrm{M}$ es un tercer cuerpo (típicamente $\mathrm{N}_{2} \mathrm{u}$ $\mathrm{O}_{2}$ ). Las fuentes dominantes del radical $\mathrm{CH}_{3} \mathrm{C}(\mathrm{O})$ $\mathrm{OO}^{*}$ son la oxidación de acetaldehído $\left(\mathrm{CH}_{3} \mathrm{CHO}\right)$, la fotólisis de acetona $\left(\mathrm{CH}_{3} \mathrm{C}(\mathrm{O}) \mathrm{CH}_{3}\right)$ y en menor grado, la fotólisis de metilglioxal $\left(\mathrm{CH}_{3} \mathrm{COCHO}\right)$ (Seinfeld y Pandis 2012):

$\mathrm{CH}_{3} \mathrm{CHO}+\mathrm{OH} \stackrel{\mathrm{O}_{2}}{\longrightarrow} \mathrm{CH}_{3} \mathrm{C}(\mathrm{O}) \mathrm{OO}^{*}+\mathrm{H}_{2} \mathrm{O}$

$\mathrm{CH}_{3} \mathrm{C}(\mathrm{O}) \mathrm{CH}_{3}+\mathrm{hv} \stackrel{\mathrm{O}_{2}}{\longrightarrow} \mathrm{CH}_{3} \mathrm{C}(\mathrm{O}) \mathrm{OO} \cdot+\mathrm{CH}_{3} \cdot$

$\mathrm{CH}_{3} \mathrm{COCHO}+\mathrm{hv} \stackrel{\mathrm{O}_{2}}{\longrightarrow} \mathrm{CH}_{3} \mathrm{C}(\mathrm{O}) \mathrm{OO} \cdot+\mathrm{HCO}$ 
Otros nitratos peroxiacilos son producidos de forma similar pero el rendimiento de su generación es mucho más bajo que la del PAN. El acetaldehído, la acetona y el metilglioxal son emitidos por fuentes primarias antrópicas y el acetaldehído y la acetona también por fuentes biogénicas, aunque también son generados en la atmósfera (de fuentes secundarias) como productos de la oxidación de COV emitidos tanto por fuentes antrópicas como biogénicas (Singh et al. 1995, Fu et al. 2018). De las fuentes primarias de emisión de acetaldehído y acetona, la combustión de gasolinas reformuladas oxigenadas y diésel es una de las más importantes (Franklin et al. 2001). Se ha propuesto que el metil ter-butil éter (MTBE) y otros como el ter-amil éter (TAME) experimentan procesos de oxidación parcial en el cilindro de combustión y en los convertidores catalíticos calientes del escape de los vehículos. Como resultado se produce la emisión de isobuteno y formaldehído como productos principales, además de otros alquenos y aldehídos como 1-buteno, acetona y acetaldehído. Por otro lado, se ha sugerido que la combustión de gas LP es una fuente importante de precursores del acetaldehído como el propeno y el 1-buteno (Warnatz 1983, Blake y Rowland 1995). Otras fuentes de emisión de propeno son las emisiones biogénicas, la industria química y ciertos vehículos a diésel con control de emisiones (Storey et al. 2005, Washenfelder et al. 2010, Rhew et al. 2017).

El acoplamiento entre las reacciones de formación de ozono y PAN muestra que en la oxidación de COV en presencia de NOx durante el periodo diurno se coproducen $\mathrm{O}_{3}$ y PAN. Numerosos reportes sobre la ocurrencia de estas dos especies en ambientes urbanos, rurales y remotos así lo demuestran (Roberts et al. 1995). Sin embargo, la tasa de coproducción de $\mathrm{O}_{3}$ y PAN depende tanto de la composición inicial de los COV como de variables meteorológicas, principalmente la radiación solar y la temperatura ambiente. Lo anterior sugiere que existe una dependencia estacional en su ocurrencia (Wunderli y Gehrig 1991, Rubio et al. 2007).

La característica más importante de la reacción de formación del PAN (reacción 8) es su dependencia con la temperatura. A temperaturas relativamente altas la reacción reversible hacia la derecha domina y el PAN regresa a sus reactivos $\mathrm{CH}_{3} \mathrm{C}(\mathrm{O}) \mathrm{OO}^{\circ}$ y $\mathrm{NO}_{2}$; este mecanismo es el principal sumidero de este peroxiacilo. De acuerdo con Altshuller (1993), a 1 atm de presión, $27^{\circ} \mathrm{C}(300 \mathrm{~K})$, y un cociente $\left[\mathrm{NO}_{2}\right] /[\mathrm{NO}]$ de entre 2 y 20 , el tiempo de vida ( $\mathrm{t}$ ) del PAN varía entre $0.75 \mathrm{~h}$ y $4.4 \mathrm{~h}$, mientras que a $7^{\circ} \mathrm{C}(280 \mathrm{~K})$, el tPAN varía entre 19 h y $112 \mathrm{~h}$. Esto es, el PAN liberará $\mathrm{NO}_{2}$ si es transportado de ambientes fríos a calientes.
Por otro lado, mientras que parte del ozono es removido por diversos mecanismos tanto químicos (reacciones con COV reactivos y NO), como por depositación seca y húmeda (remoción por estomas en las hojas de las plantas, remoción por reacciones de oxidación sobre superficies y remoción por disolución en superficies acuáticas, Monks et al. 2015), el PAN puede ser transportado durante la noche en climas fríos viento abajo, lejos de las zonas urbanas y hasta tropósferas remotas, e incluso hasta la estratósfera baja donde puede ser una fuente de radicales libres y de NOx días después de su generación, con implicaciones en la distribución regional y global del $\mathrm{O}_{3}$ y de radicales $\mathrm{OH}$ (Derwent y Jenkin 1991, Aneja et al. 1999, Fischer et al. 2014, Fadnavis et al. 2015). El PAN también puede ser producido durante la noche vía la reacción del acetaldehído con el radical nitrato. La remoción del PAN por la vegetación vía los estomas puede, bajo ciertas condiciones, ser un mecanismo adicional de remoción de PAN de tipo depositación seca (Moraveck et al. 2015).

Las primeras mediciones de PAN en la Zona Metropolitana de la Ciudad de México (ZMCM) fueron realizadas por Gaffney et al. (1999) entre febrero y marzo de 1997 con un método instrumental no comercial. En ese entonces se registró un máximo (promedio de $30 \mathrm{~min}$ ) de PAN de $34 \mathrm{ppb}_{\mathrm{v}}$ en el norte de la ciudad, en el Instituto Mexicano del Petróleo (IMP) y un promedio de los máximos diarios de 15 $\mathrm{ppb}_{\mathrm{v}}$. Los altos niveles de PAN observados en ese periodo pudieron correlacionarse con mediciones de i-buteno y formaldehído, utilizados como trazadores de emisiones vehiculares producidos principalmente por el uso de MTBE como aditivo en las gasolinas oxigenadas. No obstante, estos autores no encontraron correlación con el ozono registrado en ese sitio que alcanzó máximos de hasta $240 \mathrm{ppb}_{\mathrm{v}}$. Gaffney et al. (1999) adjudicaron los niveles de PAN a las entonces altas emisiones de alquenos y aldehídos generados por una flota vehicular que estaba utilizando gasolinas reformuladas oxigenadas sin estar equipada con convertidores catalíticos, tal y como ya se había observado por Bravo et al. (1991). Una nueva campaña de mediciones de PAN fue realizada entre abril y mayo de 2003 en el sureste de la ZMCM, en el campus Iztapalapa de la Universidad Autónoma Metropolitana (UIZ) (Marley et al. 2007). Los niveles de PAN en esta campaña presentaron un máximo de $8.5 \mathrm{ppb}_{\mathrm{v}}$ y un promedio de los máximos diarios de $3 \mathrm{ppb}_{\mathrm{v}}$, en tanto que los niveles de $\mathrm{O}_{3}$ para ese mismo sitio fueron de un máximo de $135 \mathrm{ppb}_{\mathrm{v}}$. La comparación de los valores de PAN registrados en 1997 contra los de 2003 mostraron que, si bien los sitios 
de medición y el periodo del año fueron diferentes, y que no se encontró una correlación aceptable entre PAN y $\mathrm{O}_{3}$, sí se observó una clara disminución en las razones de mezcla en ambas especies respecto a 1997. Marley et al. (2007) sugieren que los cambios en la tecnología de control de emisiones vehiculares entre 1997 y 2003 pudieron ser mayores, y que posiblemente hubo un cambio en la composición del gas propano licuado, lo que pudo llevar a una reducción importante en la emisión de los precursores orgánicos del PAN y en los niveles de $\mathrm{NO}_{2}$.

Sin embargo, a pesar de que se han establecido diversas medidas para el control del $\mathrm{O}_{3}$ en la $\mathrm{ZMCM}$ en los últimos 30 años, la disminución en el percentil 90 de los promedios horarios para cada año y el número de días por arriba de la respectiva norma de calidad del aire (95 $\mathrm{ppb}_{\mathrm{v}}$ en promedio horario) no se ha logrado en los últimos 10 años. Incluso, hubo un importante repunte en 2017, por lo que el $\mathrm{O}_{3}$ sigue siendo uno de los problemas de calidad del aire en esta zona (Velasco y Retama 2017, SEDEMA 2018a). Por otro lado, los niveles de NOx no han mostrado tendencia a la disminución desde hace casi 15 años, lo que se atribuye a la falta de controles avanzados de emisiones de estos gases en la flota vehicular a diésel en en el país y al incremento en el número de vehículos en circulación (Zavala et al. 2009, Rensoli 2017).

Por otro lado, los analizadores comerciales de NOx con métodos por quimioluminiscencia típicos de las estaciones de monitoreo reportan concentraciones sobrestimadas de $\mathrm{NO}_{2}$ debido a una interferencia inherente de estos analizadores, donde las especies de $\mathrm{NOz}\left(\mathrm{NOz}=\mathrm{PAN}+\mathrm{HNO}_{3}+\mathrm{NO}_{3}+\right.$ otros $)$, son determinadas como equivalentes a $\mathrm{NO}_{2}$, que se suma al $\mathrm{NO}_{2}$ verdadero (Winer et al. 1974, Dunlea et al. 2007). Por esta razón, al $\mathrm{NO}_{2}$ medido por el método de quimioluminiscencia se le conoce como $\mathrm{NO}_{2}$ no verdadero, aunque se aceptan estos registros como válidos para fines de evaluación de la calidad del aire (McClenny et al. 2002).

Considerando que desde hace más de 13 años no se ha vuelto a medir el PAN en la ZMCM, y que las emisiones potenciales de sus precursores no presentan un decremento formal, se propuso investigar si se han sostenido los niveles observados por Marley et al. en 2003 (Marley et al. 2007), y si ha ocurrido un cambio en la magnitud de las emisiones de COV con el acoplamiento de la química asociada a la formación de PAN y $\mathrm{O}_{3}$ entre 2003 y 2017. En este trabajo, se presenta el resultado de dos campañas cortas de monitoreo de PAN realizadas en junio y octubre de 2017 al suroeste de la ZMCM y su comparación con campañas de medición previas. Asimismo, se inves- tiga si existe una correlación entre $\mathrm{PAN}, \mathrm{O}_{3}, \mathrm{NO}_{2}$ (no verdadero) y variables meteorológicas medidas en el mismo sitio, a fin de proponer un modelo empírico para realizar un diagnóstico a largo plazo de las posibles variaciones temporales del PAN en la ZMCM.

\section{MATERIALES Y MÉTODOS}

\section{Área de estudio}

La figura 1 muestra la localización del sitio de monitoreo en el Centro de Ciencias de la Atmósfera de la UNAM en Ciudad Universitaria (CCA), al suroeste de la CdMx (19.3262 N, 99.1761 W y $2280 \mathrm{~m})$. La figura incluye la ubicación de los sitios IMP (19.489866 N, 99.145957 W, $2242 \mathrm{~m})$ y UIZ (19.360794 N, $99.07388 \mathrm{~W}, 2221 \mathrm{~m})$ donde previamente se realizaron mediciones de PAN y las rosas de viento para los periodos matutino ( $6 \mathrm{a} 12 \mathrm{~h}$ ) y vespertino (13 a $18 \mathrm{~h}$ ) obtenidas a partir de datos meteorológicos de la Red Automática de Monitoreo Atmosférico del Gobierno de la Ciudad de México (RAMA) durante las temporadas de ozono (marzo a junio) de 2008 y 2009.

\section{Métodos de medición}

Se realizaron mediciones de $\mathrm{PAN}$ y $\mathrm{NO}_{2}$ verdadero utilizando un método que combina la separación del $\mathrm{NO}_{2}$ y el PAN de la muestra por cromatografía capilar rápida para su posterior detección mediante una reacción en la interfase gas/líquido con una solución alcalina de luminol que produce quimioluminiscencia a aproximadamente $425 \mathrm{~nm}$ (GC/LCD). En este trabajo se define como $\mathrm{NO}_{2}$ verdadero a las mediciones de esta especie que no presentan la interferencia típica de los analizadores comerciales de NOx por quimioluminiscencia y que aquí son designadas como $\mathrm{NO}_{2}$ quimio. El analizador consiste de un prototipo que fue integrado siguiendo un esquema similar al sistema desarrollado por el Center for Environmental Research and Technology de la Universidad de California en Riverside (CERT-UCR; Fitz et al. 2006; Fig. 2). El método como tal, está basado en el trabajo previamente realizado por Gaffney et al. (1998) y cuya descripción detallada se presenta en Marley et al. (2004). Un resumen de la secuencia de operación del prototipo es la siguiente.

Una bomba de vacío introduce aire ambiental filtrado junto con un gas de acarreo $\left(\mathrm{N}_{2}\right.$ o aire ultrapuros) a una válvula de inyección de seis puertos (VICI-6 port valve C22-6186EH) que es controlada por un programa desarrollado por este sistema en LabView, que es un lenguaje de programación visual 

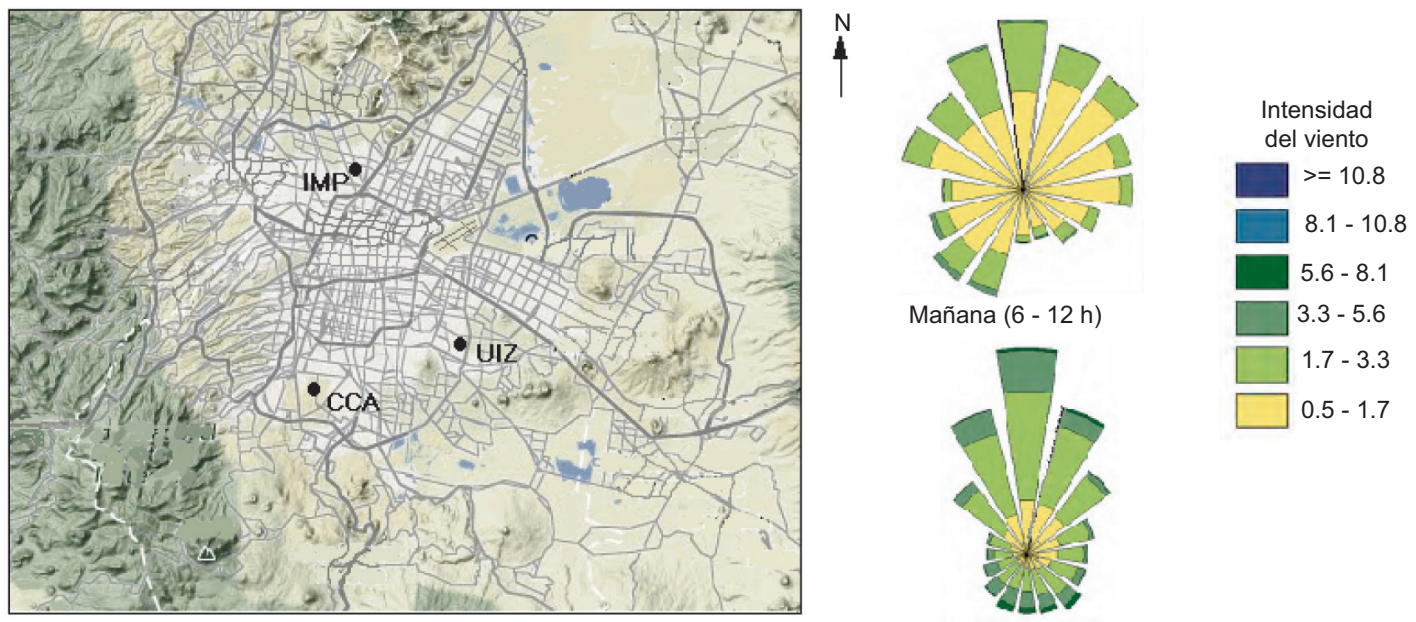

$\begin{array}{lllll}0 & 5 & 10 & 15 & 20 \\ \mathbf{k m}\end{array}$

Mañana $(6-12 \mathrm{~h})$

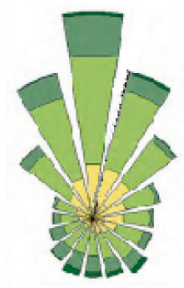

Tarde $(13-18 \mathrm{~h})$

Fig 1. Mapa de la Zona Metropolitana de la Ciudad de México mostrando la localización de los sitios IMP (19.3262 N, $99.1761 \mathrm{~W}, 2280$ m), UIZ (19.360794 N, 99.07388 W, $2221 \mathrm{~m})$ y CCA (19.3262 N, 99.1761 $\mathrm{W}, 2280 \mathrm{~m})$. Se muestran las rosas de viento para los periodos mañana $(6 \mathrm{a} 12 \mathrm{~h})$ y tarde $(13 \mathrm{a} 18 \mathrm{~h})$ para marzo-junio de 2017 de cinco estaciones de monitoreo representativas de la ZMCM. (Fuente: SEDEMA 2018b).

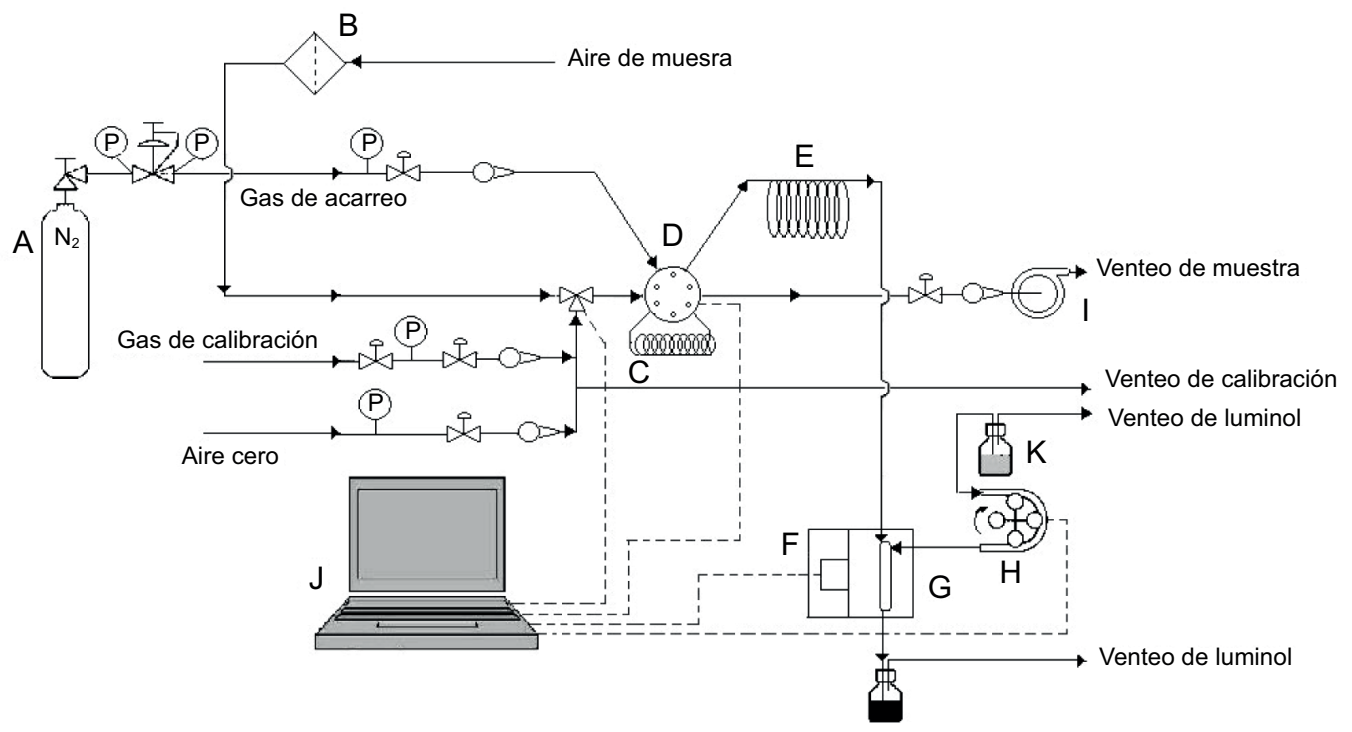

Fig 2. Diagrama del prototipo analizador $\mathrm{NO}_{2} / \mathrm{PAN}$ por cromatografía y detección por quimioluminiscencia con luminol GC/LCD. A = Tanque de gas de acarreo, $\mathrm{B}=$ Filtro de partículas en línea de muestra, $\mathrm{C}=\mathrm{Bucle}$ de muestra, $\mathrm{D}=$ Válvula de seis puertos, $\mathrm{E}=$ Columna capilar cromatográfica, $\mathrm{F}=$ Tubo fotomultiplicador, $\mathrm{G}=$ Cámara de reacción, $\mathrm{H}=$ Bomba peristáltica, $\mathrm{I}=$ Bomba de vacío, $\mathrm{J}=$ Computadora para control de válvulas y captura e integración de datos, y $\mathrm{K}=$ Reservorio de luminol fresco. (Adaptado de Fitz et al. 2006). 
gráfico compartido amablemente por D. Fitz del CERT-UCR. La muestra se inyecta, después del paso a través de una línea corta tipo rizo de PFA Teflón ${ }^{\circledR}$ de $2 \mathrm{~mL}$, a intervalos de 1 minuto a una columna cromatográfica capilar del tipo DB-1 (Agilent J\&W GC column: $5 \mathrm{~m}$ de longitud; $0.53 \mathrm{~mm}$ de diámetro y recubrimiento de $2.5 \mu \mathrm{m}$ ). La columna se mantiene a la temperatura del interior del laboratorio. Con la ayuda del gas de acarreo, el $\mathrm{NO}_{2}$ eluye primero y unos segundos después le sigue el PAN. Conforme eluyen, cada uno de ellos hace contacto sobre una gasa impregnada de luminol fresco en una cámara de reacción. La reacción entre el luminol y el $\mathrm{NO}_{2}$ y el PAN produce cierta luminosidad cuya intensidad es proporcional a la concentración de los compuestos. La intensidad de la luminosidad se registra como una señal de conteo de fotones que es enviada a la computadora para el procesamiento e integración de los respectivos picos cromatográficos. La solución amortiguadora de luminol consiste una mezcla de $0.05 \mathrm{M} \mathrm{NaOH} ; 0.1 \mathrm{M} \mathrm{Na}_{2} \mathrm{SO} 3 ; 4.5 \times 10^{-4} \mathrm{M}$ luminol (5-amino-2,3-dihidroftalazina-1,4-diona) y $0.05 \%$ $(\mathrm{v} / \mathrm{v})$ de alcohol tert-butílico en agua desionizada. El luminol gastado y el venteo del efluente gaseoso de la columna cromatográfica son drenados a una botella colectora. La sensibilidad del prototipo se ha reportado en aproximadamente $0.1 \mathrm{ppb}_{\mathrm{v}}$ (Fitz et al. 2006).

La calibración del prototipo se realiza acoplando varios procedimientos por separado, uno para calibrar el nivel de concentración máximo detectable o "span" del $\mathrm{NO}_{2}$, siendo este el primer paso, y otro para el "span" del PAN, que se realiza una vez calibrado el $\mathrm{NO}_{2}$. Para la calibración del $\mathrm{NO}_{2}$ se generan concentraciones conocidas de esta especie mediante la conversión del $\mathrm{NO}$ a $\mathrm{NO}_{2}$ por su reacción con $\mathrm{O}_{3}$ (procedimiento GPT; Gas Phase Titration) utilizando un calibrador (MCZ Mobile Calibration System Model CMK 5T) alimentado con una corriente de aire cero proveniente de un tanque de aire ultrapuro (Infra) y de NO de un tanque con una mezcla certificada de $\mathrm{NO}, \mathrm{CO}$ y $\mathrm{SO}_{2}$ en balance de $\mathrm{N}_{2}$ (Praxair®). La calibración de la línea base (cero) del prototipo se realiza alimentando directamente aire cero. El flujo de salida del calibrador es alimentado al puerto de muestra del prototipo previo a su paso por un filtro de partículas de PFA. El valor del conteo de fotones de la señal cuando se alimenta sólo aire cero equivale al ruido electrónico del tubo fotomultiplicador que es tomado por el software como la línea base o concentración de fondo. Este conteo debe restarse tanto a las lecturas durante la calibración como en la medición normal.
Para la calibración del "span" del PAN se combinaron procedimientos y recomendaciones de Gaffney et al. (1998), Fitz et al. (2006) y del manual de operación del sistema del GC/LCD del CERT-UCR (2000), en una basada en una calibración indirecta aprovechando el hecho de que los analizadores de NOx por quimioluminiscencia (NOx-CD) reportan $\mathrm{NO}_{2}$ no verdadero como previamente se mencionó y de que la reacción quimioluminiscente del luminol tanto con $\mathrm{NO}_{2}$ como al PAN es específica y estequiométricamente equivalente a cada una de estas especies

El procedimiento consiste en inyectar unos cuantos microlitros de acetaldehído líquido en una bolsa transparente de Tedlar ${ }^{\circledR}$ de 5 L previamente llenada con una corriente de gas de calibración con concentraciones conocidas de $\mathrm{NO}$ y $\mathrm{NO}_{2}$ diluidas con aire cero y un poco de aire ambiente filtrado. El aire ambiente permite introducir radicales $\mathrm{OH}$ para iniciar las reacciones. La bolsa se sella y es expuesta a luz solar directa por un tiempo de unos 30 minutos. Las reacciones que ocurren dentro de la bolsa llevan a la formación de más radicales $\mathrm{OH}$ y a la eventual formación de PAN (reacción 3), a la vez que ocurre un decaimiento en los niveles iniciales de NOx. Una vez pasado este periodo de exposición, la bolsa se coloca inmediatamente en una caja con hielo a una temperatura menor a $20^{\circ} \mathrm{C}$ para evitar la descomposición térmica del PAN y es llevada al sitio donde se ubican los analizadores. La bolsa se conecta por líneas muy cortas de tubería de PFA tanto al prototipo de GC/ LCD como al analizador de NOx-CD. La principal suposición en este procedimiento indirecto de calibración es que los NOx reportados por el analizador por quimioluminiscencia equivalen a la suma: $\mathrm{NOx}$ $=\mathrm{NO}+\mathrm{NO}_{2}$ quimio, $\mathrm{y}$ donde este último consiste de $\mathrm{NO}_{2}$ verdadero $+\mathrm{PAN}$.

Para la medición de las concentraciones ambientales de $\mathrm{PAN}$ y $\mathrm{NO}_{2}$ verdadero en términos de la razón de mezcla, la toma de muestra del prototipo de $\mathrm{NO}_{2}$ -PAN consistió de una línea de tubería PFA de aproximadamente $4 \mathrm{~m}$ de longitud y $0.65 \mathrm{~cm}$ de diámetro externo que se colocó a unos $30 \mathrm{~m}$ de distancia de la estación de monitoreo CCA de la RAMA. Las alturas de la respectiva toma de muestra fueron aproximadamente $10 \mathrm{~m}$ y $13 \mathrm{~m}$ de sobre la superficie del suelo. Para la investigación de la correlación entre PAN, ozono y $\mathrm{NO}_{2}$ se utilizaron datos de $\mathrm{O}_{3}$ y $\mathrm{NO}_{2}$ quimio reportados como promedios horarios por la estación de la RAMA (SEDEMA 2018b) durante el mismo periodo de muestreo del $\mathrm{NO}_{2}$ verdadero y el PAN del prototipo, por lo que la base de datos de éste último fue procesada para obtener los promedios horarios 
respectivos. $\mathrm{El}_{3}$ y el $\mathrm{NO}_{2}$ quimio se miden en esta estación con analizadores API modelos 400A y 200A respectivamente. Los equipos de la estación son calibrados periódicamente por personal de la RAMA.

Respecto a la información meteorológica durante las campañas, se utilizaron datos generados por la estación de la Red Universitaria de Observatorios Atmosféricos (RUOA) del CCA con los siguientes sensores: temperatura ambiente, termohigrómetro (Vaisala) HMP155a; intensidad y dirección de viento, anemómetro (Gill Instruments, Windsonic4) y radiación solar global con un piranómetro Hukseflux SR20 (RUOA-UNAM 2018). Las campañas de monitoreo abarcaron los periodos del 21 al 27 de junio y del 11 al 16 de octubre de 2017.

\section{Modelo empírico de diagnóstico}

Para la obtención del modelo empírico de diagnóstico de PAN se realizaron diversos análisis de regresión lineal entre el PAN medido y los registros de $\mathrm{O}_{3}$ y $\mathrm{NO}_{2}$ y las variables meteorológicas que se considera pueden influenciar la formación/remoción de PAN en el transcurso del día. En este caso, la selección de las variables se realizó tomando como base la experiencia que Schrimpf y colaboradores (1998) obtuvieron para una investigación similar, y en cuyo trabajo se encuentra una descripción detallada de la justificación en la aplicación de las regresiones lineales realizadas. De esta forma, la intención de obtener el modelo empírico para PAN fue combinar diversas ecuaciones de regresión lineal con coeficientes de determinación aceptables que, aplicadas selectivamente de acuerdo con el periodo del día, permitieran obtener valores de PAN a partir de variables típicamente medidas en las estaciones de monitoreo. La hipótesis de trabajo planteada fue que la coproducción de $\mathrm{O}_{3}$ y PAN en las condiciones ambientales de la ZMCM está más bajo la influencia de las variables de temperatura, radiación e intensidad de viento a lo largo de las diferentes temporadas del año, que en cambios en las emisiones, dado que en general éstas se mantienen relativamente constantes en esta escala de tiempo. De esta forma, el planteamiento del modelo empírico consideró la combinación de tres situaciones: 1) un periodo diurno de formación y acumulación de PAN a temperaturas $\leq 20^{\circ} \mathrm{C} ; 2$ ) un periodo diurno condicionado a temperaturas mayores a $20^{\circ} \mathrm{C}$ donde el PAN ya no se acumula en forma directamente proporcional a la del ozono; y 3) el periodo nocturno, donde puede haber tanto remoción como cierta formación química de PAN y acumulación que es favorecida por la estabilidad atmosférica de la noche. El modelo es contrastado contra las mediciones de las dos campañas de medición y posteriormente es aplicado al periodo conocido como temporada de ozono en la ZMCM (marzo a junio), asumiendo que el modelo empírico combinado resultante (MEC) es válido para esta temporada.

\section{RESULTADOS Y DISCUSIÓN}

\section{Campañas de medición}

La figura 3 presenta las series de tiempo de los promedios horarios de $\mathrm{PAN}, \mathrm{O}_{3}, \mathrm{NO}_{2}$ verdadero $\mathrm{y} \mathrm{NO}_{2}$ quimio, temperatura ambiente, radiación global, e intensidad y dirección de viento durante las campañas. En el cuadro I se puede observar la estadística exploratoria de las principales variables para tres periodos: campaña completa, diurno entre 10:00 y 17:00 horas (periodo de actividad fotoquímica y del incremento en la temperatura ambiente y la radiación solar) y nocturno entre las 20:00 y 06:00 horas (periodo de competencia entre remoción y formación química). Este cuadro muestra que los promedios, las medianas, los máximos y los percentiles 90 de los promedios horarios de PAN, ozono, temperatura y radiación solar fueron mayores en junio que en octubre. A fin de determinar si existió una diferencia estadística entre los registros de $\mathrm{PAN}$ y $\mathrm{O}_{3}$ entre junio y octubre durante el periodo favorable a la actividad fotoquímica y química (10:00 y 17:00 horas), se aplicó la prueba estadística t de Student a estas poblaciones de datos suponiendo varianzas desiguales (nivel de significancia $5 \%$ ). El resultado mostró que existe diferencia significativa entre los promedios de ambas especies, por lo que en principio el mes de junio es más favorable a la producción de $\mathrm{PAN} \mathrm{y}_{3}$. Es posible la combinación de los efectos de mayor temperatura y horas de insolación en junio, con respecto a la reducción en horas de insolación y de menor temperatura en octubre influyan en la química atmosférica asociada. Por comparación, el máximo reportado por Marley et al. (2004) para sus mediciones de PAN entre marzo y abril de 2003 en Iztapalapa, fue de de $8 \mathrm{ppb}_{\mathrm{v}}$, con un promedio diario máximo de $3 \mathrm{ppb}_{\mathrm{v}}$ para promedios de 30 minutos. La integración de los promedios del PAN en 30 min para las campañas de junio y octubre de 2017, resultan en máximos de 5.4 y $3.3 \mathrm{ppb}_{\mathrm{v}}$, respectivamente, y un promedio diario máximo de $1.1 \mathrm{ppb}_{\mathrm{v}}$ para todos los valores. La comparación indica que si bien el máximo para 2017 es un tanto menor al de 2003, el promedio diario máximo es incluso mayor al de 2003 reportado por Marley et al. (2004). 

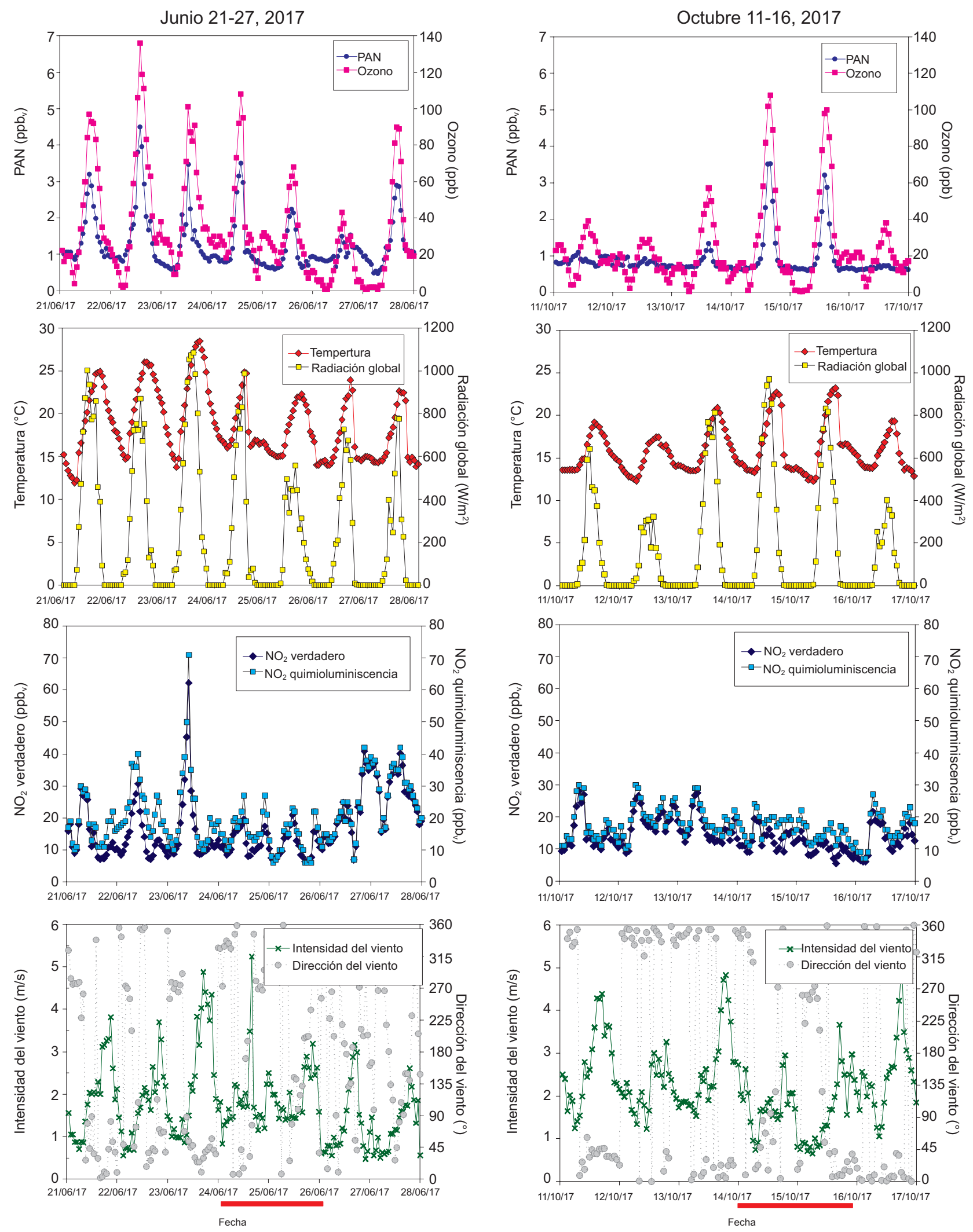

Fig 3. Series de tiempo de promedios horarios de nitrato de peroxiacetilo (PAN), ozono $\left(\mathrm{O}_{3}\right)$, temperatura ambiente ( $\left.\mathrm{T}\right)$, radiación solar global (RS), dióxido de nitrógeno medido $\left(\mathrm{NO}_{2}\right.$ verdadero), dióxido de nitrógeno medido por quimioluminiscencia $\left(\mathrm{NO}_{2}\right.$ quimio $)$ e intensidad y dirección del viento $(\mathrm{V})$ registrados al suroeste de la Ciudad de México en los periodos del 21 al 27 junio y del 11 al 17 de octubre de 2017. La línea roja indica días de fin de semana. 
CUADRO I. ESTADÍSTICA DESCRIPTIVA DE LOS PROMEDIOS HORARIOS DE NITRATO DE PEROXIACETILO (PAN), OZONO $\left(\mathrm{O}_{3}\right)$, TEMPERATURA AMBIENTE (T), RADIACIÓN TOTAL GLOBAL (RS), INTENSIDAD DEL VIENTO (V), DIÓXIDO DE NITRÓGENO MEDIDO $\left(\mathrm{NO}_{2}\right.$ verdadero $)$ y DIÓXIDO DE NITRÓGENO MEDIDO POR QUIMIOLUMINSCENCIA ( $\mathrm{NO}_{2}$ quimio), PARA EL TOTAL DE DATOS Y LOS PERIODOS DIURNO Y NOCTURNO DE LAS CAMPAÑAS DE MEDICIÓN DE JUNIO 21-27 Y OCTUBRE 11-17 DE 2017 EN EL SUROESTE DE LA ZONA METROPOLITANA DE LA CIUDAD DE MÉXICO.

\begin{tabular}{|c|c|c|c|c|c|c|c|}
\hline Descriptor & $\begin{array}{l}\text { PAN } \\
\left(p p b_{v}\right)\end{array}$ & $\begin{array}{c}\mathrm{O}_{3} \\
\left(\mathrm{ppb}_{\mathrm{v}}\right)\end{array}$ & $\begin{array}{c}\text { Temperatura } \\
\left({ }^{\circ} \mathrm{C}\right)\end{array}$ & $\begin{array}{c}\text { Radiación } \\
\text { total } \\
\left(\mathrm{W} / \mathrm{m}^{2}\right)\end{array}$ & $\begin{array}{l}\text { Intensidad } \\
\text { del viento } \\
(\mathrm{m} / \mathrm{s})\end{array}$ & $\begin{array}{c}\mathrm{NO}_{2} \\
\text { verdadero } \\
\left(\mathrm{ppb}_{\mathrm{v}}\right)\end{array}$ & $\begin{array}{c}\mathrm{NO}_{2} \\
\text { quimioluminiscencia } \\
\left(\mathrm{ppb} \mathrm{v}_{\mathrm{v}}\right)\end{array}$ \\
\hline Periodo & \multicolumn{7}{|c|}{ Campaña completa (Junio 21-27, 2017) } \\
\hline Promedio & 1.3 & 35.5 & 19.0 & 269.3 & 1.9 & 15.0 & 19.1 \\
\hline Desviación estándar & 0.8 & 28.7 & 4.0 & 318.6 & 1.0 & 8.5 & 9.4 \\
\hline Máximo & 4.5 & 136.0 & 28.5 & 1085.2 & 5.2 & 62.2 & 71.0 \\
\hline Percentil 90 & 2.2 & 83.0 & 24.8 & 783.0 & 3.2 & 25.5 & 31.4 \\
\hline Mediana & 1.0 & 27.0 & 18.0 & 73.3 & 1.7 & 11.9 & 17.1 \\
\hline Percentil 10 & 1.0 & 2.9 & 14.6 & 0.0 & 0.5 & 35.5 & 36.6 \\
\hline Periodo & \multicolumn{7}{|c|}{ Campaña completa (Octubre 11-17, 2017) } \\
\hline Promedio & 0.9 & 23.2 & 15.9 & 167.4 & 2.2 & 14.2 & 17.5 \\
\hline Desviación estándar & 0.5 & 21.3 & 2.7 & 256.1 & 0.9 & 5.0 & 4.9 \\
\hline Máximo & 3.5 & 108.0 & 23.2 & 969.2 & 5.1 & 28.0 & 30.0 \\
\hline Percentil 90 & 1.1 & 46.5 & 19.9 & 612.9 & 3.5 & 21.6 & 24.0 \\
\hline Mediana & 0.7 & 17.0 & 15.2 & 3.1 & 2.1 & 13.1 & 17.0 \\
\hline Percentil 10 & 0.6 & 11.9 & 13.0 & 0.0 & 2.0 & 13.0 & 18.3 \\
\hline Periodo & \multicolumn{7}{|c|}{ Diurno formación fotoquímica y acumulación (10:00 a 17:00 horas; Junio 21-27, 2017) } \\
\hline Promedio & 2.1 & 67.8 & 22.7 & 639.7 & 2.3 & 13.3 & 17.7 \\
\hline Desviación estándar & 0.9 & 29.7 & 2.9 & 281.5 & 0.9 & 5.9 & 6.7 \\
\hline Máximo & 4.5 & 136.0 & 28.5 & 1085.2 & 5.2 & 28.5 & 35.0 \\
\hline Percentil 90 & 3.4 & 105.5 & 26.0 & 986.8 & 3.5 & 20.9 & 26.0 \\
\hline Mediana & 1.8 & 66.5 & 22.7 & 699.0 & 2.0 & 11.3 & 16.5 \\
\hline Percentil 10 & 1.0 & 28.2 & 19.2 & 291.3 & 1.5 & 7.4 & 11.0 \\
\hline Periodo & \multicolumn{7}{|c|}{ Diurno formación fotoquímica y acumulación (10:00 a 17:00 horas; Octubre 11-17, 2017) } \\
\hline Promedio & 1.2 & 45.8 & 19.1 & 456.4 & 2.5 & 13.0 & 16.6 \\
\hline Desviación estándar & 0.8 & 25.8 & 2.2 & 269.0 & 0.9 & 3.3 & 2.9 \\
\hline Máximo & 3.5 & 108.0 & 23.2 & 969.2 & 4.4 & 21.1 & 23.0 \\
\hline Percentil 90 & 2.5 & 88.6 & 22.4 & 830.1 & 4.0 & 16.9 & 20.0 \\
\hline Mediana & 0.9 & 35.0 & 18.6 & 399.5 & 2.5 & 12.6 & 16.5 \\
\hline Percentil 10 & 0.7 & 24.1 & 16.6 & 149.4 & 1.5 & 9.4 & 13.0 \\
\hline Periodo & \multicolumn{7}{|c|}{ Nocturno (20:00 a 06:00 horas; Junio 21-27, 2017) } \\
\hline Promedio & 0.9 & 19.8 & 16.9 & 33.8 & 1.6 & 13.5 & 17.4 \\
\hline Desviación estándar & 0.2 & 10.5 & 3.0 & 32.3 & 0.9 & 7.3 & 7.7 \\
\hline Máximo & 1.3 & 41.0 & 24.8 & 79.0 & 4.3 & 40.8 & 42.0 \\
\hline Percentil 90 & 1.1 & 31.5 & 21.3 & 71.9 & 2.9 & 18.0 & 26.0 \\
\hline Mediana & 0.9 & 22.0 & 16.4 & 47.3 & 1.4 & 11.1 & 16.0 \\
\hline Percentil 10 & 0.8 & 11.5 & 15.3 & 72.5 & 1.0 & 7.5 & 11.0 \\
\hline Periodo & \multicolumn{7}{|c|}{ Nocturno (20:00 a 06:00 horas; Octubre 11-17, 2017) } \\
\hline Promedio & 0.7 & 13.7 & 14.2 & 0.3 & 2.1 & 13.0 & 16.2 \\
\hline Desviación estándar & 0.1 & 6.2 & 1.1 & 2.7 & 0.7 & 4.3 & 4.6 \\
\hline Máximo & 1.0 & 26.0 & 17.4 & 21.9 & 4.2 & 23.7 & 26.0 \\
\hline Percentil 90 & 0.9 & 21.5 & 15.9 & 0.0 & 2.8 & 18.6 & 21.5 \\
\hline Mediana & 0.7 & 13.5 & 13.9 & 0.0 & 2.1 & 12.5 & 16.0 \\
\hline Percentil 10 & 0.6 & 4.0 & 13.7 & 4.9 & 1.2 & 9.6 & 13.0 \\
\hline
\end{tabular}


Para el periodo nocturno, las medianas de PAN y $\mathrm{O}_{3}$ fueron 0.9 y $0.7 \mathrm{ppb}_{\mathrm{v}}$ y, 22.0 y $13.5 \mathrm{ppb}_{\mathrm{v}}$, para junio y octubre respectivamente y los correspondientes percentiles 90 fueron 1.1 y $0.9 \mathrm{ppb}_{\mathrm{v}}$ para el PAN, y para el $\mathrm{O}_{3}$ de 31.5 y $21.5 \mathrm{ppb}_{\mathrm{v}}$. Los valores indican que se mantiene cierto nivel de concentración de fondo relativamente constante para el PAN independientemente de la temporada del año por ser estable a bajas temperaturas, mientras que el $\mathrm{O}_{3}$ presenta niveles por debajo de su concentración de fondo de $\sim 40 \mathrm{ppb}_{\mathrm{v}}$, lo que refleja la intensidad de la reacción de titulación con NO. Este efecto es más intenso en octubre posiblemente porque la capa de inversión nocturna está más restringida en su altura llevando a una mayor acumulación de NO nocturno. Los niveles del PAN nocturno se ubican dentro del rango observado por Gaffney et al. (1999) y Marley et al. (2004) en el orden de $\sim 1 \mathrm{ppb}_{\mathrm{v}}$.

Con respecto al $\mathrm{NO}_{2}$ verdadero sus promedios horarios no difirieron mucho entre junio y octubre aún en los periodos diurno y nocturno (13.0 a $16.3 \mathrm{ppb}_{\mathrm{v}}$ ), aunque los máximos alcanzaron valores mayores en octubre $\left(62.2 \mathrm{ppb}_{\mathrm{v}}\right)$. Asimismo, la comparación entre el $\mathrm{NO}_{2}$ medido por $\mathrm{GC} / \mathrm{LCD}$ con respecto al $\mathrm{NO}_{2}$ quimio medido por el método quimioluminiscente indica que las mediciones del instrumento utilizando el segundo método excedieron consistentemente la concentración promedio horaria del $\mathrm{NO}_{2}$ verdadero.

CUADRO II. RESULTADOS DELANÁLISIS DE CORRELACIÓN ENTRE LOS PROMEDIOS HORARIOS DE NITRATO DE PEROXIACETILO (PAN) Y LAS VARIABLES: OZONO $\left(\mathrm{O}_{3}\right)$, TEMPERATURA (T), RADIACIÓN TOTAL GLOBAL (RS), E INTENSIDAD DEL VIENTO (V) REGISTRADOS EN LAS CAMPAÑAS DE MONITOREO DE JUNIO Y OCTUBRE DE 2017 PARA EL PERIODO DIURNO CON MAYOR COEFICIENTE DE DETERMINACIÓN $\left(\mathrm{R}^{2}\right)^{\mathrm{a}}$.

\begin{tabular}{|c|c|c|}
\hline Correlación & Ecuación resultante & Condición de ajuste \\
\hline \multicolumn{3}{|c|}{21 a 27 de junio, 2017} \\
\hline Ozono vs. PAN & $\begin{array}{l}\mathrm{PAN}=0.700 e^{\left(0.0143^{\prime} \mathrm{O} 3\right)} \\
\mathrm{R}^{2}=0.82\end{array}$ & $\begin{array}{l}\text { PAN }=0.6785 e^{\left(0.0146^{\prime} \mathrm{O} 3\right)} \\
\mathrm{R}^{2}=0.77 \\
\mathrm{~T}>20^{\circ} \mathrm{C}\end{array}$ \\
\hline Temperatura vs. PAN & $\begin{array}{l}\mathrm{PAN}=0.2561 e^{\left(0.0819^{\prime} \mathrm{T}\right)} \\
\mathrm{R}^{2}=0.41\end{array}$ & $\begin{array}{l}\text { PAN }=0.0566 e^{\left(0.1628^{\prime} \mathrm{T}\right)} \\
\mathrm{R}^{2}=0.72 \\
08: 00-13: 00 \text { horas }\end{array}$ \\
\hline Radiación vs. PAN & $\begin{array}{l}\mathrm{PAN}=0.8715 e^{\left(0.0010^{\prime} \mathrm{RS}\right)} \\
\mathrm{R}^{2}=0.39\end{array}$ & $\begin{array}{l}\mathrm{PAN}=0.6032 e^{\left(0.0014^{\prime} \mathrm{RS}\right)} \\
\mathrm{R}^{2}=0.60 \\
07: 00-13: 00 \text { horas }\end{array}$ \\
\hline Intensidad viento vs. PAN & $\begin{array}{l}\text { PAN }=1.1575 e^{\left(-0.094^{\prime} \mathrm{V}\right)} \\
\mathrm{R}^{2}=0.031\end{array}$ & $\begin{array}{l}\text { PAN }=3.1362 e^{\left(-0.1779^{\prime} \mathrm{V}\right)} \\
\mathrm{R}^{2}=0.13 \\
12: 00-17: 00 \text { horas }\end{array}$ \\
\hline \multicolumn{3}{|c|}{11 a 17 de octubre, 2017} \\
\hline Ozono vs. PAN & $\begin{array}{l}\mathrm{PAN}=0.5689 e^{\left(0.0148^{\prime} \mathrm{O} 3\right)} \\
\mathrm{R}^{2}=0.83\end{array}$ & $\begin{array}{l}\mathrm{PAN}=0.4021 e^{\left(0.0203^{\prime} \mathrm{O} 3\right)} \\
\mathrm{R}^{2}=0.94 \\
\mathrm{~T}>20^{\circ} \mathrm{C}\end{array}$ \\
\hline Temperatura vs. PAN & $\begin{array}{l}\mathrm{PAN}=0.1267 e^{\left(0.1137^{\prime} \mathrm{T}\right)} \\
\mathrm{R}^{2}=0.58\end{array}$ & $\begin{array}{l}\mathrm{PAN}=0.0566 e^{\left(0.1047^{\prime} \mathrm{T}\right)} \\
\mathrm{R}^{2}=0.56 \\
08: 00-13: 00 \text { horas }\end{array}$ \\
\hline Radiación vs. PAN & $\begin{array}{l}\mathrm{PAN}=0.742 e^{\left(0.006^{\prime} \mathrm{RS}\right)} \\
\mathrm{R}^{2}=0.39\end{array}$ & $\begin{array}{l}\mathrm{PAN}=0.65 e^{\left(0.0005^{\prime} \mathrm{RS}\right)} \\
\mathrm{R}^{2}=0.31 \\
07: 00-13: 00 \text { horas }\end{array}$ \\
\hline Intensidad viento vs. PAN & $\begin{array}{l}\mathrm{PAN}=1.051 e^{\left(-0.0631^{\prime} \mathrm{V}\right)} \\
\mathrm{R}^{2}=0.023\end{array}$ & $\begin{array}{l}\text { PAN }=3.1362 e^{\left(-0.4546^{\prime} \mathrm{V}\right)} \\
\mathrm{R}^{2}=0.55 \\
12: 00-17: 00 \text { horas }\end{array}$ \\
\hline
\end{tabular}

${ }^{\mathrm{a}}$ Las unidades de las ecuaciones son: PAN (ppb), $\mathrm{O}_{3}(\mathrm{ppb}), \mathrm{T}\left({ }^{\circ} \mathrm{C}\right), \mathrm{RS}\left(\mathrm{W} / \mathrm{m}^{2}\right), \mathrm{y} \mathrm{V}(\mathrm{m} / \mathrm{s})$. 
La diferencia porcentual entre los dos tipos de mediciones para todos los registros varió en un rango de entre $22 \%$ y $28 \%$ entre junio y octubre, mientras que esta misma diferencia en los valores máximos fue de entre el 7 y el $11 \%$, respectivamente. Dunlea et al. (2007) observaron esta misma problemática analítica en 2006 en la Ciudad de México. La diferencia resulta de la interferencia positiva que especies como el PAN y otras especies oxidadas de nitrógeno diferentes a los $\mathrm{NOx}$ tienen en los analizadores de $\mathrm{NO}_{2}$ que utilizan el método quimioluminiscente.

Cabe mencionar que el efecto fin de semana, en el que se esperaría que por disminuir las emisiones de NOx por la reducción del tráfico vehicular habría una reducción tanto del PAN como del $\mathrm{O}_{3}$, ésta no se observa claramente. Incluso durante octubre ambos contaminantes tuvieron niveles elevados en sábado y domingo, lo que es de esperarse en ambientes donde el ozono es sensible a los COV y en particular a aldehídos (Torres-Jardón et al. 2007).

\section{Correlación empírica}

El cuadro II presenta las ecuaciones de regresión lineal resultantes entre el PAN y cada una de las variables estudiadas para los diferentes periodos analizados y que tuvieron la mejor correlación de acuerdo al valor del coeficiente de determinación (R2). Conforme a lo esperado, el PAN tiene una buena correlación con el ozono (rango de R2 entre 0.7 y 0.9 ), siendo más alto el coeficiente $\mathrm{R} 2$ durante octubre y más bajo en junio. La pérdida de correlación entre el PAN y el ozono en junio se asocia con el hecho de que la temperatura ambiente es más alta en este mes que en octubre. Al separar por periodos, la temperatura y la radiación presentan una correlación positiva moderada de la mañana hasta poco después del mediodía, mientras que la intensidad de viento muestra una ligera tendencia a perder la linealidad con el PAN por la tarde, lo cual se aprecia por el valor bajo del coeficiente de determinación observado para ese periodo del día.

En junio las variables de temperatura y radiación mostraron cierto grado de asociación con el PAN, mientras que el viento no, en tanto que en octubre, sólo la temperatura y el viento parecen asociarse, aunque con valores bajos del coeficiente $\mathrm{R}^{2}$. Este resultado sugiere que las diferencias estacionales en la temperatura, la radiación solar y la intensidad del viento podrían ser incluidas en correlaciones lineales múltiples junto con el ozono en un modelo empírico combinado para obtener las concentraciones aproximadas de PAN para otros periodos del año.

Algunos investigadores han utilizado la correlación entre los máximos de PAN y ozono para obtener los valores esperados de PAN conociendo los valores de ozono (Rubio et al. 2007). Sin embargo, para las dos campañas realizadas sólo se cuenta con 14 pares de valores de máximos de PAN y ozono, lo que podría dar por resultado una correlación con alta incertidumbre. Con el fin de que el modelo empírico permita aproximar las concentraciones del PAN a largo plazo, se realizó un análisis de varias correlaciones lineales múltiples con base en los resultados antes mostrados. Para esto, se utilizó el conjunto de datos de las mediciones del PAN con el prototipo y las otras variables medidas por la estación de la RUOA y la de la RAMA en el Centro de Ciencias de la Atmósfera a lo largo de las 24 horas en las dos campañas realizadas. Las ecuaciones de regresión lineal múltiple resultantes y el esquema conceptual de trabajo que presentaron los mejores resultados se muestran en el cuadro III, mientras que la comparación entre el PAN medido con el obtenido con el modelo empírico integrado para cada una de las campañas se presenta en la figura 4. Esta figura muestra que el modelo empírico combinado (MEC) reproduce razonablemente los valores medidos de PAN sin importar la temporalidad en el año. La ecuación de correlación simple entre el PAN modelado y el PAN medido, incluyendo los periodos diurno y nocturno, resulta en: [PAN modelo] $=1.003 \times[\mathrm{PAN}$ medido $] ; \mathrm{R}^{2}=0.875$.

Asumiendo que el MEC permitiría obtener el comportamiento teórico esperado del PAN en un periodo del año diferente con altos niveles de ozono, se recabaron datos de los niveles de ozono y $\mathrm{NO}_{2}$ quimio de la estación CCA de la RAMA y de temperatura, radiación global e intensidad de viento de la estación CCA de la RUOA para los meses de abril, mayo y junio de 2017, que es cuando se presentan las así llamadas contingencias ambientales. Las series de tiempo de los resultados de la modelación del PAN y la de los registros de ozono, temperatura ambiente y radiación global del trimestre seleccionado se presentan en la figura 5.

La modelación del PAN muestra que la asociación de este oxidante con $\mathrm{O}_{3}$ y la temperatura ambiente es definitiva y que a altas concentraciones de ozono, hay también altas concentraciones de PAN. Sin embargo, no se observa un incremento significativo del PAN durante la ocurrencia de los eventos de ozono mayores a $150 \mathrm{ppb}_{\mathrm{v}}$ incluso ni cuando se registró contingencia por $\mathrm{O}_{3}$ en la ZMCM (23 de mayo de 2017). Es posible que dadas las altas temperaturas de ese mes, y en particular el día de la contingencia $\left(27^{\circ} \mathrm{C}\right)$, el valor del PAN simulado resultó moderado a pesar que el valor del ozono fue de $185 \mathrm{ppb}_{\mathrm{v}}$. La comparación de las series de tiempo entre el PAN 
CUADRO III. ECUACIONES DE CORRELACIÓN LINEAL MÚLTIPLE Y SUS RESPECTIVOS COEFICIENTES DE DETERMINACIÓN $\left(\mathrm{R}^{2}\right)$ OBTENIDAS A PARTIR DE LAS MEDICIONES DE NITRATO DE PEROXIACETILO (PAN) POR CROMATOGRAFÍA DE GASES RÁPIDA CON DETECCIÓN DE QUIMIOLUMINISCENCIA DE LUMINOL (GC/LCD) Y LOS REGISTROS DE OZONO $\left(\mathrm{O}_{3}\right)$, TEMPERATURA (T), RADIACIÓN TOTAL GLOBAL (RS), INTENSIDAD DEL VIENTO (V) Y DIÓXIDO DE NITRÓGENO MEDIDO POR QUIMIOLUMINISCENCIA (NO 2quimio ) DURANTE LAS CAMPAÑAS DE MONITOREO DE JUNIO Y OCTUBRE DE 2017. LAS ECUACIONES FUERON OBTENIDAS A UN NIVEL DE CONFIANZA DEL $95 \%{ }^{\mathrm{a}}$.

\begin{tabular}{|c|c|c|}
\hline Periodo del día & Ecuación & Comentario conceptual \\
\hline $\begin{array}{l}09: 00-19: 00 \text { horas } \\
\text { Para } \mathrm{T}<20^{\circ} \mathrm{C}\end{array}$ & $\begin{array}{l}\mathrm{PAN}=1.1898+\left(0.03052 \times \mathrm{O}_{3}\right) \\
-(0.0378 \times \mathrm{T})-(0.000137 \times \mathrm{RS}) \\
-(0.15018 \times \mathrm{V}) \\
\mathrm{R}^{2} \text { ajustada }=0.88\end{array}$ & $\begin{array}{l}\text { Se asume que la temperatura, la radiación solar y } \\
\text { el viento influyen en la coproducción de PAN y } \\
\text { ozono cuando la temperatura ambiente es menor } \\
\text { a } 20^{\circ} \mathrm{C} \text {. Por arriba de esta condición inicia la } \\
\text { descomposición térmica del PAN. }\end{array}$ \\
\hline $\begin{array}{l}\text { 13:00 - 19:00 horas } \\
\text { Para } \mathrm{T}>20^{\circ} \mathrm{C}\end{array}$ & $\begin{array}{l}\mathrm{PAN}=2.65739537+\left(0.030563 \times \mathrm{O}_{3}\right) \\
-(0.13698 \times \mathrm{T})+(0.03091 \times \mathrm{V}) \\
+\left(0.004724 \times \mathrm{NO}_{2} \text { quimio }\right) \\
\mathrm{R}^{2} \text { ajustada }=0.91\end{array}$ & $\begin{array}{l}\text { Por arriba de } \mathrm{T}=20^{\circ} \mathrm{C} \text { y después de las } 13: 00 \\
\text { horas, ocurre la descomposición del PAN y la } \\
\text { correlación con } \mathrm{O}_{3} \text { cambia. La RS va en caída } \\
\text { y es posible que el viento tenga influencia tanto } \\
\text { en el acarreo advectivo de masas de aire al sitio } \\
\text { de monitoreo como de su dispersión. Se incluyó } \\
\text { al } \mathrm{NO}_{2} \text { por quimioluminiscencia como factor de } \\
\text { ajuste empírico, toda vez que su medición incluye } \\
\text { al PAN encubierto y es la medición disponible } \\
\text { para otros periodos. }\end{array}$ \\
\hline $\begin{array}{l}19: 00-24: 00 \text { horas } \\
y \\
1: 00-09: 00 \text { horas }\end{array}$ & $\begin{array}{l}\mathrm{PAN}=0.63775 \times e^{\left(0.01229 \times \mathrm{NO}_{2} \text { quimio }\right)} \\
\mathrm{R}^{2}=0.756\end{array}$ & $\begin{array}{l}\text { Durante la noche no hay producción fotoquímica } \\
\text { y la ruta de formación de } \mathrm{PAN} \text { es a partir de la } \\
\text { reacción entre acetileno y } \mathrm{NO}_{2} \text { tanto del producido } \\
\text { por la titulación }{ }^{\mathrm{b}} \text { de } \mathrm{O}_{3} \text { con } \mathrm{NO} \text { y por emisiones } \\
\text { frescas de } \mathrm{NO}_{2} \text {. Temperaturas menores a } 20^{\circ} \mathrm{C} \\
\text { mantienen los niveles del PAN remanente del } \\
\text { día. La correlación con } \mathrm{NO}_{2} \text { quimio se utiliza como } \\
\text { trazador integrado de tanto las concentraciones de } \\
\text { PAN, como del } \mathrm{NO}_{2} \text { disponible para su formación. }\end{array}$ \\
\hline
\end{tabular}

a Las unidades son: PAN (ppb), $\mathrm{O}_{3}(\mathrm{ppb}), \mathrm{T}\left({ }^{\circ} \mathrm{C}\right), \mathrm{RS}\left(\mathrm{W} / \mathrm{m}^{2}\right), \mathrm{V}(\mathrm{m} / \mathrm{s})$ y $\mathrm{NO}_{2}$ quimio $(\mathrm{ppb})$.

${ }^{b}$ La reacción de titulación es: $\mathrm{NO}+\mathrm{O}_{3} \rightarrow \mathrm{NO}_{2}+\mathrm{O}_{2}$.
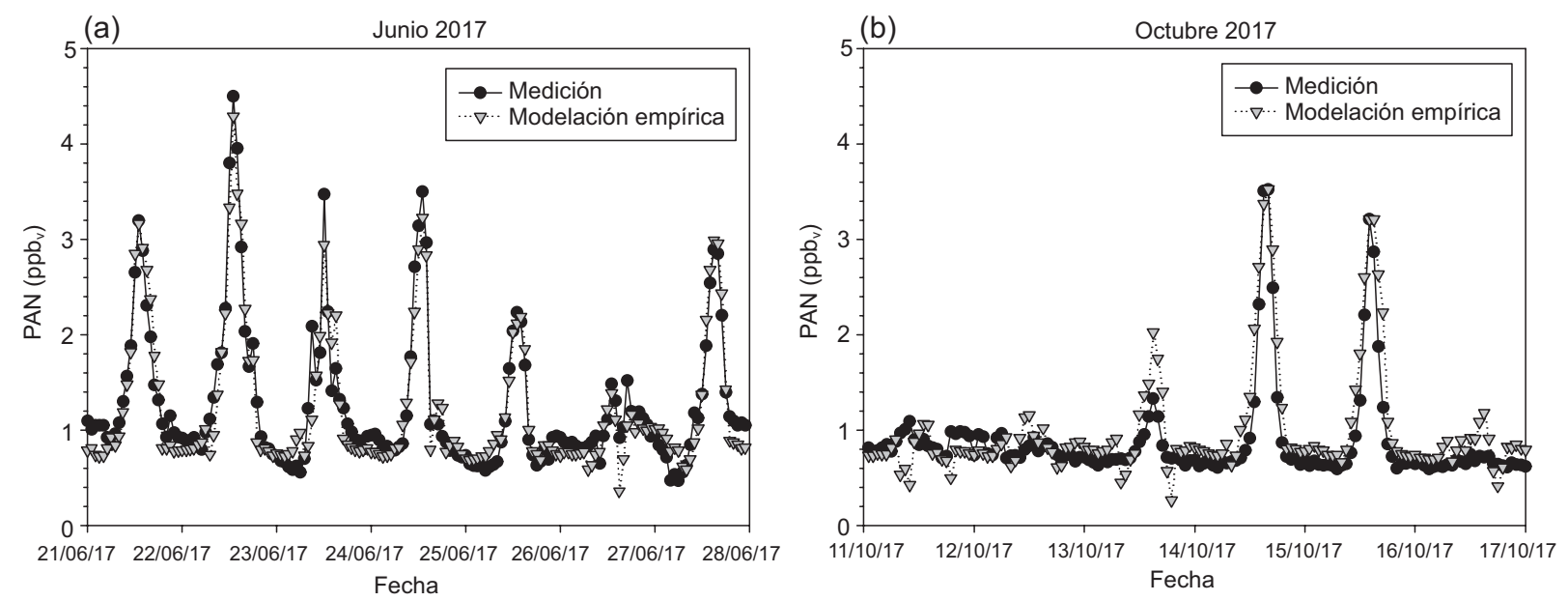

Fig. 4. Series de tiempo de promedios horarios de nitrato de peroxiacetilo (PAN) modelado y medido para los periodos: (a) 21 a 27 de junio, y (b) 11 a 17 de octubre de 2017. 
y el ozono con la de la radiación solar indica que esta variable meteorológica no presenta gran correspondencia con estos contaminantes. El cuadro IV

(a)

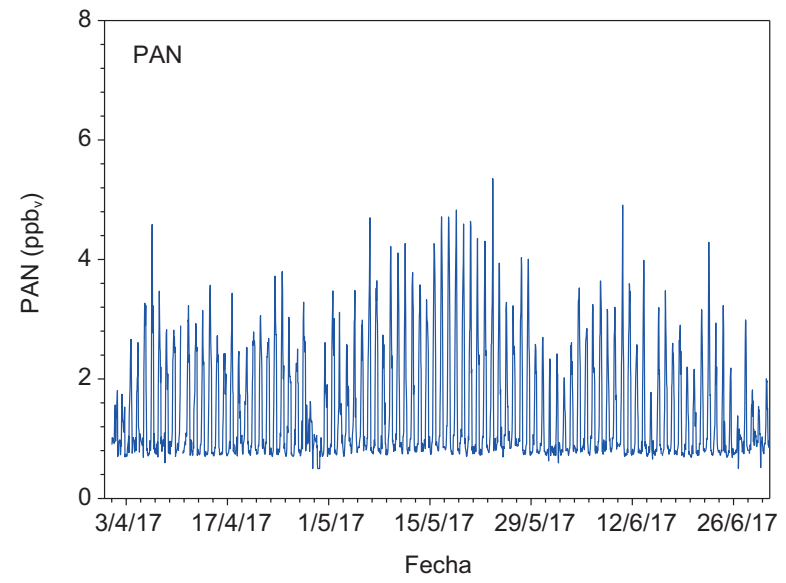

(c)

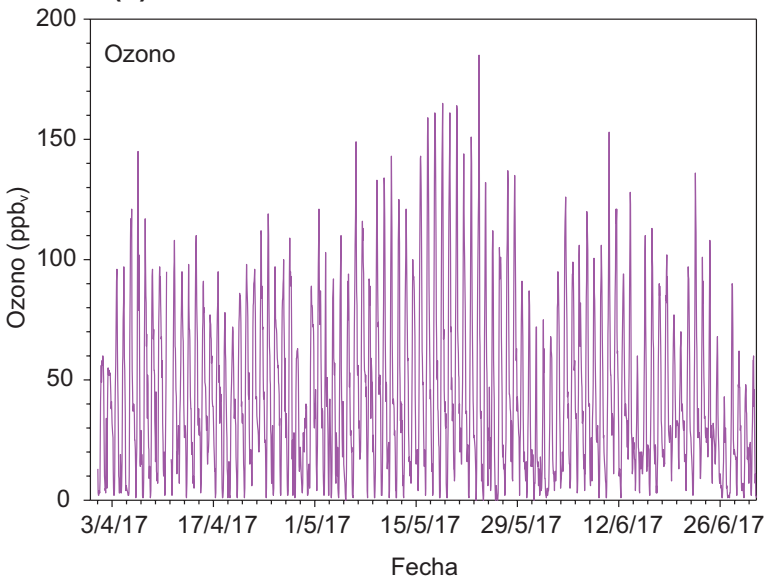

muestra la estadística descriptiva del PAN modelado y las variables meteorológicas utilizadas en la simulación.

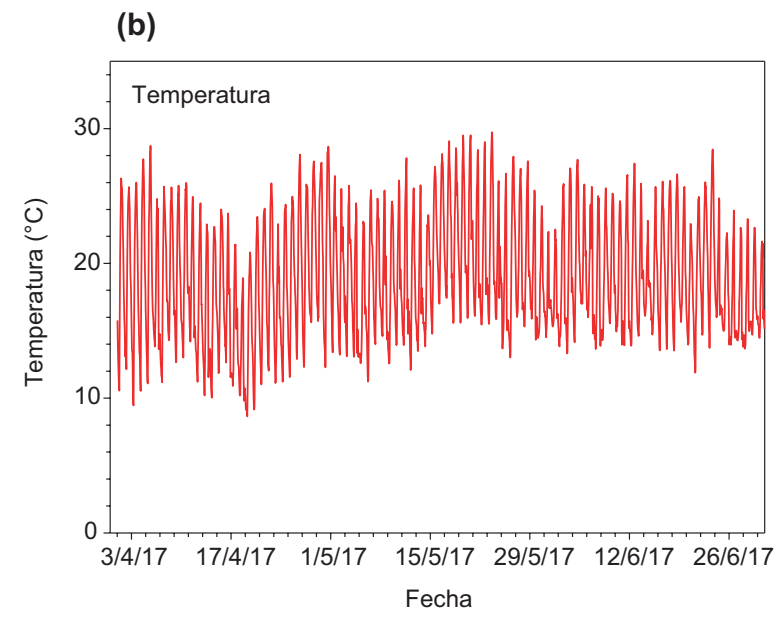

(d)

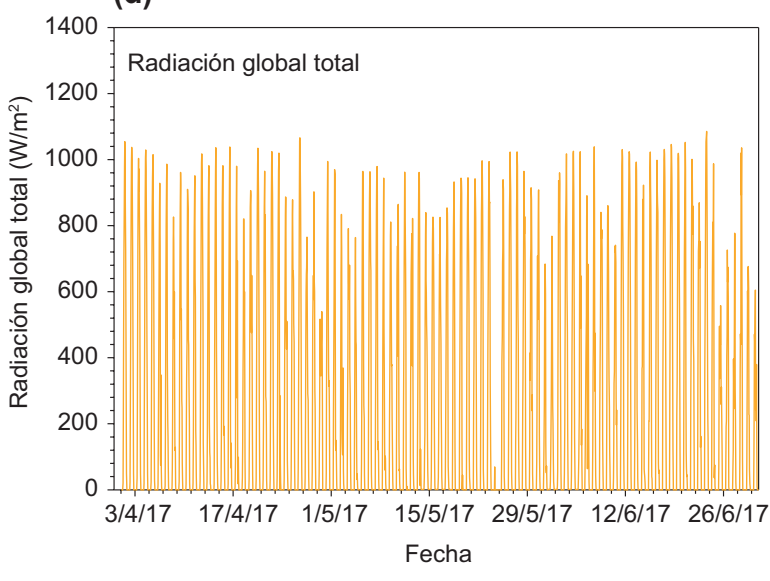

Fig 5. Series de tiempo de la concentración teórica simulada de nitrato de peroxiacetilo (PAN) con el modelo empírico combinado y las respectivas series de tiempo de ozono $\left(\mathrm{O}_{3}\right)$, temperatura ambiente $(\mathrm{T})$ y radiación global total (GS) registradas al suroeste de la Zona Metropolitana de la Ciudad de México entre el $1^{\circ}$ de abril y el 30 de junio de 2017.

CUADRO IV. ESTADÍSTICA DESCRIPTIVA DE LOS PROMEDIOS HORARIOS SIMULADOS DE NITRATO DE PEROXIACETILO (PAN) Y LOS PROMEDIOS HORARIOS DE OZONO $\left(\mathrm{O}_{3}\right)$, TEMPERATURA (T), RADIACIÓN GLOBAL TOTAL (RS), INTENSIDAD DEL VIENTO (V), Y DIÓXIDO DE NITRÓGENO MEDIDO POR QUIMIOLUMINISCENCIA ( $\mathrm{NO}_{2}$ quimio), PARA EL PERIODO ABRIL1 A JUNIO 30 DE 2017 EN EL SUROESTE DE LA ZONA METROPOLITANA DE LA CIUDAD DE MÉXICO.

\begin{tabular}{llccccc}
\hline Descriptor & $\begin{array}{c}\mathrm{PAN} \\
\left(\mathrm{ppb}_{\mathrm{v}}\right)\end{array}$ & $\begin{array}{c}\mathrm{O}_{3} \\
\left(\mathrm{ppb}_{\mathrm{v}}\right)\end{array}$ & $\begin{array}{c}\text { Temperatura } \\
\left({ }^{\circ} \mathrm{C}\right)\end{array}$ & $\begin{array}{c}\text { Radiación total } \\
\left(\mathrm{w} / \mathrm{m}^{2}\right)\end{array}$ & $\begin{array}{c}\text { Intensidad del } \\
\text { viento }(\mathrm{m} / \mathrm{s})\end{array}$ & $\begin{array}{c}\mathrm{NO}_{2} \text { verdadero } \\
\left(\mathrm{ppb}_{\mathrm{v}}\right)\end{array}$ \\
\hline Promedio & 1.43 & 43.4 & 19 & 246.2 & 1.82 & 21.74 \\
Desviación estándar & 0.88 & 34.9 & 4.42 & 335.22 & 0.98 & 10.74 \\
Máximo & 5.35 & 185 & 2973 & 1085.2 & 6.39 & 73 \\
Percentil 90 & 2.8 & 93 & 25.33 & 840.14 & 3.19 & 36.01 \\
Mediana & 0.96 & 34 & 18.39 & 12.35 & 1.61 & 19 \\
Percentil 10 & 0.75 & 5 & 13.78 & 0 & 0.785 & 11 \\
\hline
\end{tabular}


CUADRO V. RESUMEN DE NIVELES DE LA RAZÓN DE MEZCLA DEL NITRATO DE PEROXIACETILO (PAN) REGISTRADAS EN OTRAS ZONAS URBANAS DEL MUNDO Y EL SUROESTE DE LA CIUDAD DE MÉXICO.

\begin{tabular}{|c|c|c|c|c|c|}
\hline Año & Ciudad & $\begin{array}{l}\text { Periodo de } \\
\text { estudio }\end{array}$ & $\begin{array}{l}\text { Razón de mezcla } \\
\text { máxima }\left(p p b_{v}\right)\end{array}$ & 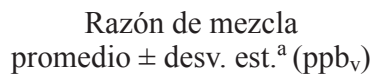 & Referencia \\
\hline 1997 & Ciudad de México & Febrero-marzo & 34 & 15 & Gaffney et al. 1999 \\
\hline 2003 & Ciudad de México & Abril-mayo & 8 & 3 & Marley et al. 2007 \\
\hline 2003 & Santiago de Chile & Enero & 22 & 6.4 & Rubio et al. 2004 \\
\hline 2006 & Pekin, China & Agosto & 11.22 & 1.95 & Zhang et al. 2011 \\
\hline \multirow[t]{2}{*}{$2007-2008$} & \multirow{2}{*}{ Roma, Italia } & Invierno & 7.3 & 2.1 & \multirow{2}{*}{$\begin{array}{l}\text { Movassaghi } \\
\text { et al. } 2010\end{array}$} \\
\hline & & Verano & 30.3 & 5.7 & \\
\hline 2010 & Beijing, China & Enero-marzo & 3.51 & 0.7 & Zhang et al. 2014 \\
\hline 2011 & Seúl, Corea del Sur & Enero-diciembre & 5.03 & 0.64 & Lee et al. 2013 \\
\hline $2015-2016$ & Jinan, China & Noviembre-enero & 9.61 & $1.89 \pm 1.42$ & Liu et al. 2018 \\
\hline 2016 & Jinan, China & Abril-julio & 13.47 & $2.54 \pm 1.44$ & Liu et al. 2018 \\
\hline \multirow[t]{3}{*}{2017} & \multirow{3}{*}{$\begin{array}{l}\text { Ciudad de } \\
\text { México }\end{array}$} & $\begin{array}{l}\text { Junio } \\
\text { (medición) }\end{array}$ & 4.5 & $1.3 \pm 0.8$ & \multirow{3}{*}{ Este estudio } \\
\hline & & $\begin{array}{l}\text { Octubre } \\
\text { (medición) }\end{array}$ & 3.5 & $0.9 \pm 0.5$ & \\
\hline & & $\begin{array}{l}\text { Abril-junio } \\
\text { (modelación } \\
\text { empírica) }\end{array}$ & 5.35 & $1.43 \pm 0.88$ & \\
\hline
\end{tabular}

${ }^{a}$ Se incluye la desviación estándar cuando el dato está disponible.

El máximo simulado del PAN alcanza un promedio horario de $5.3 \mathrm{ppb}_{\mathrm{v}}$ y resulta asociado al máximo del ozono de $185 \mathrm{ppb}_{\mathrm{v}}$. Al igual que el máximo medido en la campaña de junio, este máximo de PAN simulado es también menor al máximo reportado por Marley et al. (2004) en 2003 en Iztapalapa, mientras que el promedio de toda la campaña es casi la mitad del promedio diario máximo reportado por estos autores. Una comparación de los valores de PAN reportados para otras partes del mundo con los obtenidos en este estudio se presenta en el cuadro $\mathbf{V}$.

De acuerdo con los datos del cuadro $\mathbf{V}$, mientras que los máximos del PAN en el suroeste de la Ciudad de México se acercan a los casos de Beijing en 2010 y de Seúl, Corea del Sur en 2011, los promedios globales son muy cercanos a los reportados en Jinan, China. El reporte más reciente para Jinan, de mediciones realizadas en el invierno de 2015-2016, indica que el promedio del PAN en la Ciudad de México está ligeramente debajo del de esa población. Jinan, con poco más de 7 millones de habitantes, presenta similitudes con la ZMCM en cuanto a que está parcialmente encerrada por montañas y sufre severos problemas de contaminación del aire, particularmente de esmog fotoquímico (Zhang et al. 2017). Esto sugiere que aún y cuando los niveles tanto del PAN como del ozono en la ZMCM disminuyeron notoriamente entre 1997 y 2003, la reducción de sus concentraciones promedio, las medianas y los precentiles 90 de los horarios entre este último año y 2017 no ha sido significativa (Velazco y Retama 2017, Molina et al. 2019).

Una probable causa de la disminución aparentemente desigual en los niveles de $\mathrm{PAN} \mathrm{y}_{3}$ entre 2003 y 2017 puede ser el cambio en la magnitud de las emisiones de los COV precursores de ambas especies. Con respecto al acetaldehído, las mediciones disponibles más recientes para la ZMCM son para el año 2007 (Báez et al. 2008). La comparación de estas mediciones que corresponden al periodo diurno (08:00 a 20:00 horas) con equivalentes para el año 2001 entre marzo y mayo para el centro urbano y el suroeste de la ZMCM fueron de $2.8 \mathrm{ppb}_{\mathrm{v}}$ y $2.6 \mathrm{ppb}_{\mathrm{v}}(2001)$, y de $6.0 \mathrm{ppb}_{\mathrm{v}}$ y 5.0 $\mathrm{ppb}_{\mathrm{v}}$ (2007), respectivamente (Báez et al. 2008). Los datos indican que los niveles de acetaldehído mostraron un incremento. 
En contraste, la comparación de la suma de los niveles de compuestos aromáticos importantes (benceno, tolueno, etilbenceno, m, y p-xileno, o-xileno, 1,2,4-trimetil benceno) con la suma de los niveles de olefinas representativas (eteno, propeno, 1-buteno, 1,3-butadieno, trans-2-buteno, cis-2-buteno e isopreno) medidos en los mismos sitios que para el acetaldehído de la zona urbana (centro y suroeste) para el periodo de 6:00 a 9:00 horas entre abril y mayo de diferentes años, muestra una reducción importante en los aromáticos, pero un claro incremento en las olefinas (JaimesPalomera et al. 2017). Para los años 2003 y 2012, en el sitio centro, las sumas de las olefinas fueron $35.6 \mathrm{ppb}_{\mathrm{v}}$ y $39.7 \mathrm{ppb}_{\mathrm{v}}$, y en el suroeste de 18.1 $\mathrm{ppb}_{\mathrm{v}}$ y $21.4 \mathrm{ppb}_{\mathrm{v}}$, respectivamente. En tanto que para los aromáticos, las sumas fueron $37.0 \mathrm{ppb}_{\mathrm{v}}$ y $14.7 \mathrm{ppb}_{\mathrm{v}}$ para el centro y, $12.6 \mathrm{ppb}_{\mathrm{v}}$ y $5.4 \mathrm{ppb}_{\mathrm{v}}$ para el suroeste, respectivamente. Es factible que la reducción en el potencial de formación de ozono de los aromáticos se haya compensado con un incremento en ese mismo potencial de las olefinas. En comparación, los niveles de 1-buteno fueron de $3.9 \mathrm{ppb}_{\mathrm{v}}$ en 2003 y $2.4 \mathrm{ppb}_{\mathrm{v}}$ en 2012 en La Merced (Velasco et al. 2007, Jaimes-Palomera 2017). En Beijing, China, en 2011 se reportó un rango entre 0.1 y $1 \mathrm{ppb}_{\mathrm{v}}$ de 1-buteno (Wang et al. 2014), en Roma, Italia, un promedio de $0.41 \mathrm{ppb}_{\mathrm{v}}$ para 2011 (Fanizaa et al. 2014) y en Santiago de Chile un promedio de $1.18 \mathrm{ppb}_{\mathrm{v}}$ en 2010 (Toro et al. 2014). Claramente se aprecia que la emisión de precursores de PAN sigue siendo importante. Por otro lado, si bien tanto las olefinas como varios aromáticos son precursores de acetaldehído, la reducción en los niveles de aromáticos pudo llevar a una reducción en la producción fotoquímica de acetaldehído. Sin embargo, esta producción secundaria de acetaldehído se compensa por su emisión primaria asociada al uso de aditivos oxigenados en las gasolinas distribuidas en la zona metropolitana, lo cuál podría justificar el hecho de que entre 2003 y 2017 los niveles de ozono no presenten una reducción clara. Asimismo, el PAN muestra sólo una ligera reducción con respecto a los niveles del promedio de los máximos reportados por Marley et al. (2007) para 2003, como se discutió previamente.

Dado que se siguen presentando niveles altos de $\mathrm{O}_{3}$ por arriba de la norma de calidad del aire actual, y como existe una coproducción asociada con el PAN, ambos siguen siendo un problema potencial de salud pública en el centro de México, aunque no con la intensidad de en años previos.

\section{CONCLUSIONES}

Se midió nitrato de peroxiacetilo (PAN) en dos campañas cortas de monitoreo en junio y octubre de 2017 en un sitio al suroeste de la ZMCM. Para la medición se utilizó un analizador prototipo basado en cromatografía de gases para la separación de PAN y NO2 y su posterior detección por luminol. Los promedios horarios de la razón de mezcla para el PAN fueron de $1.3 \mathrm{ppbv}$ en junio y 0.9 ppbv en octubre de 2017, con máximos de 0.5 y 3.5 ppbv, respectivamente. Los valores medidos del PAN fueron ligeramente más bajos que los reportados en 2003 en esta zona metropolitana. Se identifica una clara coproducción de ozono y PAN la cual a su vez se identifica como consecuencia de los altos niveles de sus precursores comunes (acetaldehído y 1-buteno). Tanto el PAN como el ozono muestran un patrón diurno con máximos a media tarde y mínimos en la noche. Se identifica la influencia de las condiciones meteorológicas entre los meses de junio y octubre en los niveles del PAN registrado.

Se obtuvo un modelo lineal múltiple empírico de diagnóstico de los niveles del PAN en ausencia de sus mediciones utilizando los registros del PAN (variable dependiente) y del ozono, NO2 medido por el método de quimioluminiscencia, temperatura ambiente, radiación solar e intensidad del viento (variables independientes). El modelo combina varias ecuaciones de regresión lineal para tres periodos del día: 1) periodo diurno de formación y acumulación de PAN; 2) periodo diurno condicionado a temperaturas mayores a $20{ }^{\circ} \mathrm{C}$ donde el PAN ya no se acumula en forma directamente proporcional a la del ozono; y 3 ) periodo nocturno de sostenimiento parcial del PAN remanente por estabilidad atmosférica a bajas temperaturas. El modelo empírico resultante reproduce razonablemente los niveles del PAN medidos en las dos campañas de medición y es utilizado para inferir las razones de mezcla del PAN que pudieron haberse registrado en la temporada de ozono de 2017 (abril-junio) en la Ciudad de México, que incluyó un evento de contingencia atmosférica.

La fuerte correlación encontrada entre el ozono y el PAN indica la persistencia de daño potencial de ambos contaminantes fotoquímicos a la salud humana y a la vegetación dentro de la megalópolis de la ZMCM. Es necesario redirigir las políticas ambientales hacia un mejor control en las emisiones de los precursores de O3 y PAN a fin de reducir su impacto potencial. 


\section{AGRADECIMIENTOS}

Norma I. Carrasco-Mijarez agradece el apoyo del Consejo Nacional de Ciencia y Tecnología (CONACyT) por la beca otorgada para cursar el doctorado en el Programa de Posgrado en Ciencias de la Tierra dentro del programa de doctorado de la Universidad Nacional Autónoma México y al apoyo económico para adquirir el analizador utilizado en el desarrollo de este estudio otorgado a través del proyecto "Evaluación de las correlaciones entre $\mathrm{O}_{3}$ y $\mathrm{NO}_{\text {y }}$ y su implicación como indicadores fotoquímicos en la evaluación de control de ozono en la Ciudad de México" (CONACyT clave 52201). También agradecemos al Dr. Luis Gerardo Ruiz Suárez por las facilidades en el uso del equipo instrumental del Grupo Fisicoquímica Atmosférica del Centro de Ciencias de la Atmósfera de la Universidad Nacional Autónoma de México y a Luis Miguel Barbosa Pérez Taylor por su apoyo en la calibración y operación del prototipo. El estudio es una extensión del proyecto "Evaluación de las correlaciones entre $\mathrm{O}_{3}$ y $\mathrm{NO}_{\text {y }}$ y su implicación como indicadores fotoquímicos en la evaluación de control de ozono en la Ciudad de México" (CONACyT clave 52201).

\section{REFERENCIAS}

Altshuller A. P. (1993). PANs in the atmosphere. J. Air Waste Manag. 43 (9), 1221-1230. https://doi.org/10.1 080/1073161X.1993.10467199

Aneja V. P., Harstsell B. E., Kim D. S. y Grosjean D. (1999). Peroxyacetyl nitrate in Atlanta, Georgia: comparison and analysis of ambient data for suburban and downtown locations. J. Air Waste Manag. 49 (2), 177184. https://doi.org/10.1080/10473289.1999.10463786

Báez A. P., Torres M. C., García R., Padilla H. y Belmont R. (2008). Measurement of carbonyls in three urban zones of the Mexico City Metropolitan Area and one rural zone. Open Atmos. Sci. J. 2, 61-67. https://doi. org/10.2174/1874282300802010061

Blake D. R. y Rowland F. S. (1995). Urban leakage of liquefied petroleum gas and itsimpact on Mexico City air quality. Science 269 (5226), 953-956. https://doi. org/10.1126/science.269.5226.953

Bravo H. A., Torres R. J. y Sosa R. E. (1991). Motor vehicle pollution control in Mexico City. Memorias. International Fuels and Lubricants Meeting and Exposition. Toronto, Canada. 7 al 10 de octubre, 1991. En línea para reimpresión https://saemobilus.sae.org/ content/912426/

CERT-UCR (2000). Standard operating procedure routine operation of $\mathrm{NO}_{2} / \mathrm{PAN} \mathrm{GC}$ for CCOS, Center for
Environmental Research and Technology. Manual. Riverside, California, EUA, 22 pp.

Derwent R. G. y Jenkin M. E. (1991). Hydrocarbons and the long range transport of ozone and PAN across Europe. Atmos. Environ. A-Gen. 25A, 1661-1678. https:// doi.org/10.1016/0960-1686(91)90025-3

Dunlea E. J., Herndon S. C., Nelson D. D., Volkamer R. M., San Martini F., Sheehy P. M., Zahniser M. S., Shorter J. H., Wormhoudt J. C., Lamb B. K., Allwine E. J., Gaffney J. S., Marley N. A., Grutter M., Marquez C., Blanco S., Cardenas B., Retama A., Ramos Villegas C. R., Kolb C. E., Molina L. T. y Molina M. J. (2007). Evaluation of nitrogen dioxide chemiluminescence monitors in a polluted urban environmental. Atmos. Chem. Phys. 7, 2691-2704. https://doi.org/10.5194/ acp-7-2691-2007

Fadnavis S., Semeniuk K., Schultz M. G., Kiefer M., Mahajan A., Pozzoli L. y Sonbawane S. (2015). Transport pathways of peroxyacetyl nitrate in the upper troposphere and lower stratosphere from different monsoon systems during the summer monsoon season. Atmos. Chem. Phys. 15 (20), 11477-11499. https://doi.org/10.5194/acp-15-11477-2015

Fanizza C., Incoronato F., Baiguera S., Schiro R. y Brocco D. (2014). Volatile organic compound levels at one site in Rome urban air. Atmos. Pollut. Res. 5 (2), 303-314. https://doi.org/10.5094/APR.2014.036

Fischer E. V., Jacob D. J., Yantosca R. M., Sulprizio M. P., Millet D. B., Mao J., Paulot F., Singh H. B., Roiger A., Ries L., Talbot R. W., Dzepina K. y Pandey Deolal S. (2014). Atmospheric peroxyacetyl nitrate (PAN): A global budget and source attribution. Atmos. Chem. Phys. 14, 2679-2698. https://doi.org/10.5194/acp-142679-2014

Fitz D., Pankratz D., Bumiler K. y Smith M. (2006). Measurement of $\mathrm{NO}_{2}$ and PAN by gas chromatography with luminol detection. Memorias. Air and Waste Management 99th Annual Conference and Exhibition. Nueva Orleans, Luisiana, EUA. 20 de junio, 2006. 1600-1609 pp. CD-ROM.

Franklin P. M., Koshland C. P., Lucas D. y Sawyer R. F. (2001). Evaluation of combustion by-products of MTBE as a component of reformulated gasoline. Chemosphere 42, 861-872. https://doi.org/10.1016/ S0045-6535(00)00261-7

Fu T. M., Jacob D. J., Wittrock F., Burrows J. P., Vrekoussis M. y Henze D. K. (2008). Global budgets of atmospheric glyoxal and methylglyoxal, and implications for formation of secondary organic aerosols. J. Geophys. Res-Atmos. 113 (D15). https://doi. org/10.1029/2007JD009505

Gaffney J. S., Bornick R. M., Chen Y. H. y Marley N. A. (1998). Capillary gaschromatographic analysis of 
nitrogen dioxide and PANs with luminolchemiluminescent detection. Atmos. Environ. 32 (8), 1445-1454. https://doi.org/10.1016/S1352-2310(97)00098-8

Gaffney J. S., Marley N. A., Cunningham M. M. y Doskey P. V. (1999). Measurements of peroxyacyl nitrates (PANs) in Mexico City: Implications for mega city air qualityimpacts on regional scales. Atmos. Environ. 33, 50035012. https://doi.org/10.1016/S1352-2310(99)00263-0

Jaimes-Palomera M., Retama A., Elias-Castro G., NeriaHernández A., Rivera-Hernández O. y Velasco E. (2016). Non-methane hydrocarbons in the atmosphere of Mexico City: Results of the 2012 ozone-season campaign. Atmos. Environ. 132, 258-275. https://doi. org/10.1016/j.atmosenv.2016.02.047

Jaimes M. (2017). Diseño del monitoreo de compuestos precursores de ozono en la atmósfera de la Ciudad de México y su área metropolitana. Tesis de Doctorado. Facultad de Ingeniería, Universidad Nacional Autónoma de México. Ciudad de México, México, 127 pp.

Kourtidis K. A., Fabian P., Zerefos C. y Rappenglück B. (1993). Peroxyacetyl nitrate (PAN), peroxypropionyl nitrate (PPN) and PAN/ozone ratio measurements at three sites in Germany. Tellus B 45 (5), 442-457. https:// doi.org/10.1034/J.1600-0889.1993.t01-3-00004.X

Lee J. B., Yoon J. S., Jung K., Eom S. W., Chae Y. Z., Cho S. J., Sohn J. R., Kim S. D. y Kim K. H. (2013). Peroxyacetyl nitrate (PAN) in the urban atmosphere. Chemosphere 93 (9), 1796-1803. https://doi.org/10.1016/j. chemosphere.2013.06.019

Liu L., Wang X., Chen J., Xue L., Wang W., Wen L., Li D. y Chen T. (2018). Understanding unusually high levels of peroxyacetyl nitrate (PAN) in winter in urban Jinan, China. J. Environ. Sci. 71, 249-260. https://doi. org/10.1016/j.jes.2018.05.015

Marley N. A., Gaffney J. S., White R. V., RodriguezCuadra L., Herndon S. E., Dunlea E. y Molina M. J. (2004). Fast gas chromatography with luminolchemiluminescence detection for the simultaneous determination of nitrogendioxide and peroxyacetyl nitrate in the atmosphere. Rev. Sci. Instrum. 75 (11), 4595-4605. https://doi.org/10.1063/1.1805271

Marley N. A., Gaffney J. S., Ramos-Villegas R. y Cárdenas-González B. (2007).Comparison of measurements of peroxyacyl nitrates and primary carbonaceousaerosol concentrations in Mexico City determined in 1997 and 2003. Atmos. Chem.Phys. 7, 2277-2285. https:// doi.org/10.5194/acp-7-2277-2007

McClenny W. A., Williams E. J., Cohen R. C. y Stutz J. (2002). Preparing to measure the effects of the NOx SIP call-methods for ambient air monitoring of NO, $\mathrm{NO}_{2}$, NOy, and individual $\mathrm{NOz}$ species. J. Air Waste Manag. Assoc. 52 (5), 542-562. https://doi.org/10.10 80/10473289.2002.10470801
Molina L. T., Velasco E., Retama A. y Zavala M. (2019). Experience from integrated air quality management in the Mexico City Metropolitan Area and Singapore. Atmosphere 10 (9), 512. https://doi.org/10.3390/ atmos 10090512

Monks P. S., Archibald A. T., Colette A., Cooper O., Coyle M., Derwent R., Fowler D., Granier C., Law K. S., Mills G. E., Stevenson D. S., Tarasova O., Thouret V., Von Schneidemesser E., Sommariva R., Wild O. y Williams M. L. (2015). Tropospheric ozone and its precursors from the urban to the global scale from air quality to short-lived climate forcer. Atmos. Chem. Phys. 15, 8889-8973. https://doi.org/10.5194/acp-15-8889-2015

Moravek A., Stella P. y Foken I. (2015). Influence of local air pollution on the deposition of peroxyacetyl nitrate to a nutrient-poor natural grassland ecosystem. Atmos. Chem. Phys. 15 (2), 899-911. https://doi.org/10.5194/ acp-15-899-2015

Movassaghi K., Russo M. V. y Avino P. (2012) The determination and role ofperoxyacetil nitrate in photochemical processes in atmosphere. Chem. Cent. J. 6, S8. https://doi.org/10.1186/1752-153X-6-S2-S8

NARSTO (2000). An assessment of tropospheric ozone pollution - A North American perspective. North American Research Strategy for Tropospheric Ozone [en línea]. https://www.narsto.org/sites/narsto-dev. ornl.gov/files/OAChapter3.pdf 17/05/2019

Rappenglück B., Kourtidis K. y Fabian P. (1993). Measurement of ozone andperoxyacetylnitrate (PAN) in $\mathrm{Mu}-$ nich. Atmos. Environ. B-Urb. 27B, 293-305. https:// doi.org/10.1016/0957-1272(93)90024-Z

Rappenglück B., Oyola P., Olaeta I. y Fabian P. (2000). The evolution of photochemical smog in the Metropolitan Area of Santiago de Chile. J. Appl. Meteor. 39 (3), 275-290. https://doi.org/10.1175/15200450(2000)03 9<0275:TEOPSI $>2.0 . \mathrm{CO} ; 2$

Rensoli S. R. (2017). Análisis de la tendencia de las concentraciones de $\mathrm{NO}, \mathrm{NO}_{2}, \mathrm{O}_{3}$ y $\mathrm{NOx}$ en el periodo 2000-2015 en la Zona Metropolitana de la Ciudad de México y su implicación en la química atmosférica local. Tesis de Licenciatura. Facultad de Química, Universidad Nacional Autónoma de México. Ciudad de México, México, 206 pp.

Rhew R. C., Deventer M. J., Turnipseed A. A., Warneke C., Ortega J., Shen S. y Smith J. N. (2017). Ethene, propene, butene and isoprene emissions from a ponderosa pine forest measured by relaxed eddy accumulation. Atmos. Chem. Phys. 17 (21), 13417. https://doi. org/10.5194/acp-17-13417-2017

Roberts J. M., Tanner R. L., Newman L., Bowersox V. C., Bottenheim J. W., Anlauf K. G., Brice K. A., Parrish D. D., Fehsenfeld F. C., Buhr M. P. y Meagher J. F.(1995). Relationships between PAN 
and ozone at sites in eastern North America. J. Geophys. Res-Atmos. 100 (D11), 22821-22830. DOI:10.1029/95jD01221

Rubio M. A., Oyola P., Gramsch E., Lissi E., Pizarro J. y Villena G. (2004). Ozone and peroxyacetylnitrate in downtown Santiago, Chile. Atmos. Environ. 38 (29), 4931-4939. https://doi.org/10.1016/j.atmosenv.2004.0.051

Rubio M. A., Gramsch E., Lissi E. y Villena G. (2007). Seasonal dependence ofperoxyacetylnitrate (PAN) concentrations in downtown Santiago, Chile. Atmósfera 20 (4), 319-328.

RUOA-UNAM (2018). Observatorio Atmosférico UNAM. Red Universitaria deObservatorios Atmosféricos de la Universidad Nacional Autónoma de México. Base de datos de calidad del aire de la Ciudad de México [en línea]. http://www.aire.cdmx.gob.mx/default. php?opc=\%27aqBhnmQ=\%27 15/11/2017

Schrimpf W., Linaerts K., Müller K. P., Koppmann R. K. y Rudolph J. (1998).Peroxyacetyl nitrate (PAN) measurements during the POPCORN campaign. J. Atmos. Chem. 3, 139-159. https://doi. org/10.1023/A:1006004031055

SEDEMA (2018a). 6a glosa informe de Gobierno. Secretaría del Medio Ambiente de la Ciudad de México. Informe. Ciudad de México, México, 700 pp.

SEDEMA (2018b). Datos derivados del monitoreo atmosférico. Calidad del Aire. Secretaría del Medio Ambiente de la Ciudad de México. Bases de datos. Ciudad de México, México [en línea]. http://www.aire. cdmx.gob.mx/default.php?opc $=\% 27 \mathrm{aKBhnmM=027}$ 01/17/2018

Seinfeld J. H. y Pandis S. N. (2012). Atmospheric chemistry and physics: from air pollution to climate Change. $2^{\text {nd }}$ ed. John Wiley y Sons, Nueva York, EUA, 1232 pp.

Singh H. B. (1987). Reactive nitrogen in the troposphere. Environ. Sci. Technol. 21 (4),320-327. https://doi. org/10.1021/es00158a001

Singh H. B., Kanakidou M., Crutzen P. J. y Jacob D. J. (1995). High concentrations and photochemical fate of oxygenated hydrocarbons in the global troposphere. Nature378, 50-54. https://doi.org/10.1038/378050a0

Storey M. J., Lewis Sr., A. S., West H. B., Huff P. Sh., Sluder Sc. C., Wagner M. R., Domingo N., Thomas J. y Kass M. (2005). Hydrocarbon species in the exhaust of diesel engines equipped with advanced emissions control devices. Fuels, Engines, and Emissions Research Center. Oak Ridge, Tennesse, EUA [en línea]. http:// crcsite.wpengine.com/wp-content/uploads/2019/05/ AVFL-10b-2-Final-Report-January-31-2005.pdf $01 / 27 / 2018$

Toro R. A., Donoso C. S., Seguel R. A., Morales R. G. y Leiva M. A. (2014). Photochemical ozone pollution in the Valparaiso Region, Chile. Air Qual. Atmos. Health. 7 (1), 1-11. https://doi.org/10.1007/s11869-013-0218-7

Torres-Jardón R., García-Reynoso A., Jazcilevich-Diamant A. y Ruiz-Suárez L. G. (2007). El cociente HCHO/ NOy como indicador fotoquímico potencial de la sensibilidad $\mathrm{O}_{3}-\mathrm{NO}-\mathrm{COV}$ s en la ZMCM. Memorias. VI Simposio de Contaminación. Ciudad de México, México, 17 al 19 de Abril, 2007, pp. 110-116.

Velasco E., Lamb B., Westberg H., Allwine E., Sosa G., Arriaga-Colina J. L., Jobson B. T., Alexander M. L., Prazeller P., Knighton W. B., Rogers T. M., Grutter M., Herndon S. C., Kolb C. E., Zavala M., de Foy B., Volkamer R., Molina L. T. y Molina M. J. (2007). Distribution, magnitudes, reactivities, ratios and diurnal patterns of volatile organic compounds in the Valley of Mexico during the MCMA 2002 and 2003 field campaigns. Atmos. Chem. Phys. 7, 329-353. https:// doi.org/10.5194/acp-7-329-2007

Velasco E. y Retama A. (2017). Ozone's threat hits back Mexico city. Sustain. Cities Soc. 31, 260-263. https:// doi.org/10.1016/j.scs.2016.12.015

Warnatz J. (1983). The mechanism of high temperature combustion of propane andbutane. Combust. Sci. Technol. 34 (1-6), 177-200. https://doi. org/10.1080/00102208308923692

Washenfelder R. A., Trainer M., Frost G. J., Ryerson T. B., Atlas E. L., De Gouw J. A. y Peischl J. (2010). Characterization of $\mathrm{NOx}, \mathrm{SO}_{2}$, ethene, and propene from industrial emission sources in Houston, Texas. J. Geophys. Res-Atmos. 115 (D16). https://doi. org/10.1029/2009JD013645

Winer A. M., Peters J. W., Smith J. P. y Pitts J. N. Jr. (1974). Response of commercial chemiluminescent $\mathrm{NO}-\mathrm{NO}_{2}$ analyzers to other nitrogen-containing compounds. Environ. Sci. Technol. 8 (13), 1118-1121. https://doi. org/10.1021/es60098a004

Wunderli S. y Gehrig R. (1991). Influence of temperature on formation and stability of surface PAN and ozone. A two year field study in Switzerland. Atmos. Environ. A-Gen. 25A, 1599-1608. https://doi.org/10.1016/09601686(91)90018-3

Zhang J. B., Xu Z., Yang G. y Wang B. (2011). Peroxyacetyl nitrate (PAN) and peroxypropionyl nitrate (PPN) in urban and suburban atmospheres of Beijing, China. Atmos. Chem. Phys. Discuss. 11 (3), 8173-8206. https://doi.org/10.5194/acpd-11-8173-2011

Zhang H., Xu X., Lin W. y Wang Y. (2014). Wintertime peroxyacetyl nitrate (PAN) in the megacity Beijing: Role of photochemical and meteorological processes. J. Environ. Sci. 26 (1), 83-96. https://doi.org/10.1016/ S1001-0742(13)60384-8

Zhang J., Liu Y., Cui L., Liu Sh., Yin X. y Li H. (2017). Ambient air pollution, smogepisodes and mortality 
in Jinan, China. Sci. Rep-Uk. 7 (1), 1-8. https://doi. org/10.1038/s41598-017-11338-2

Zavala A., Herndon S. C., Wood E. C., Onasch T. B., Knighton W. B., Marr L. C.,KolbC. E. y Molina L. T. (2009). Evaluation of mobile emissionsmea- surementscontributions to Mexico City's emissions inventory using on-road and cross-roademissions measurements and ambient data. Atmos. Chem. Phys. 9(17), 6305-6317. https://doi.org/10.5194/ acp-9-6305-2009 